Bull. Soc. math. France

132 (1), 2004, p. 105-136

\title{
FILLING RADIUS AND SHORT CLOSED GEODESICS OF THE 2-SPHERE
}

\author{
By StÉphane Sabourau
}

\begin{abstract}
We show that the length of the shortest nontrivial curve among the simple closed geodesics of index zero or one and the figure-eight geodesics of null index provides a lower bound on the area and the diameter of the Riemannian 2-spheres.

RÉSumÉ (Rayon de remplissage et courtes géodésiques fermées de la 2-sphère)

Nous montrons que la longueur de la plus courte courbe non triviale parmi les géodésiques simples fermées d'indice zéro ou un et les géodésiques en huit d'indice nul fournit une minoration sur l'aire et le diamètre des deux-sphères riemanniennes.
\end{abstract}

\section{Introduction}

Let $M$ be a closed connected smooth Riemannian manifold of dimension $n$. The Riemannian metric $g$ on $M$ induces a distance $d_{g}$ on $M$.

The map $i:\left(M, d_{g}\right) \hookrightarrow\left(L^{\infty}(M),\|\|.\right)$ defined by $i(x)()=.\operatorname{dist}_{M}(x,$.$) is an$ embedding from the metric space $\left(M, d_{g}\right)$ into the Banach space $L^{\infty}(M)$ of all bounded functions on $M$ with the sup-norm $\|$.$\| . This natural embedding$ is a (strong) isometry between metric spaces, i.e., it preserves the distances. Note that Riemannian embeddings of closed manifolds into Euclidean spaces are not isometric in this sense. Considering $M$ isometrically embedded in the

Texte reçu le 9 octobre 2002, accepté le 10 janvier 2003

Stéphane Sabourau, Laboratoire de Mathématiques et Physique Théorique, Université de Tours, Parc de Grandmont, 37200 Tours (France)

E-mail : sabourau@gargan.math.univ-tours.fr

2000 Mathematics Subject Classification. - 53C20.

Key words and phrases. - Filling radius, closed geodesics, 1-cycles.

BUlletin DE LA SOCiÉtÉ MATHÉMATiQUE DE FRANCE 
Banach space $L^{\infty}(M)$, we define $U_{\delta}(M)$ as the $\delta$-tubular neighborhood of $M$ in $L^{\infty}(M)$. The homology coefficients will be in $\mathbb{Z}$, if $M$ is orientable, and in $\mathbb{Z}_{2}$, otherwise.

Definition. - The filling radius of $M$, denoted $\operatorname{FillRad}(M)$, is the infimum of positive reals $\delta$ such that $\left(i_{\delta}\right)_{*}([M])=0 \in H_{n}\left(U_{\delta}(M)\right)$, where $i_{\delta}: M \hookrightarrow U_{\delta}(M)$ is the inclusion and $[M] \in H_{n}(M)$ is the fundamental class of $M$.

In this paper, we show the following curvature free estimate.

TheOrem 1.1. - Let $M$ be a Riemannian 2-sphere, then

$$
\operatorname{FillRad}(M) \geq \frac{1}{12} \operatorname{scg}(M)
$$

where $\operatorname{scg}(M)$ denotes the length of the shortest nontrivial closed geodesic on $M$.

This statement admits a stronger version in which occur the Morse index and the number of self-intersection points of closed geodesics. Note, however, that the constant involved is not as good as in the first version.

Main Theorem 1.2. - Let $M$ be a Riemannian 2-sphere, then

$$
\operatorname{FillRad}(M) \geq \frac{1}{20} \bar{L}(M)
$$

where $\bar{L}(M)$ is the length of the shortest nontrivial curve among the simple closed geodesics of index zero or one and the figure-eight geodesics of null index.

For metrics all of whose geodesics are non-degenerate (bumpy metrics) the same inequality holds if we replace $\bar{L}(M)$ by $L(M)$, where $L(M)$ represents the length of the shortest nontrivial curve among the simple closed geodesics of index 1 and the figure-eight geodesics of null index.

Some examples illustrating the different cases of the Main Theorem are presented in this paper (see Remark 4.10).

Before going further, let us review some known results to which these inequalities are related.

These two theorems extend to the simply connected case some filling radius estimates related to the 1-dimensional systole.

The 1-dimensional systole of a non-simply connected closed Riemannian manifold $(M, g)$ is defined as the infimum of the lengths of noncontractible closed curves. This lower bound, denoted $\operatorname{sys}_{1}(M, g)$, is attained by the length of a closed geodesic.

In [17] (see also [7], [18] and [19]), M. Gromov showed that every essential manifold of dimension $n$ satisfies the isosystolic inequality

$$
\operatorname{Vol}(M, g) \geq C_{n} \operatorname{sys}_{1}(M, g)^{n}
$$

where $C_{n}$ is a positive constant depending only on $n$.

TOME $132-2004-\mathrm{N}^{\mathrm{O}} 1$ 
In the above statement, whose converse was established in [3], a closed manifold $M$ is said to be essential if there is a map $f$ from $M$ to a $K(\pi, 1)$ space such that $f_{*}([M]) \neq 0$. In particular, $T^{n}, \mathbb{R} P^{n}$ and all closed aspherical manifolds are essential.

Isosystolic inequalities on surfaces were previously established in [33], [1], [8], $[9$, p. 43] and [21]. An inequality similar to (1.3) also holds for the stable systole under suitable topological conditions (see [19], [17], [22], [5], [4] and [24]).

Examples of non-essential manifolds with "long" systole and "small" volume can easily be constructed. The product metric on $S^{1} \times S^{2}$ where the length of $S^{1}$ is long and the area of $S^{2}$ is small provides such an example. However, they may still have a short contractible closed geodesic whose length is bounded from above in terms of the volume.

For Riemannian 2-spheres, C. Croke showed in [12] that

$$
\operatorname{Area}(M) \geq \frac{1}{(31)^{2}} \operatorname{scg}(M)^{2}, \quad \operatorname{Diam}(M) \geq \frac{1}{9} \operatorname{scg}(M) .
$$

It is unknown whether or not the length of the shortest nontrivial closed geodesic provides a lower bound on the volume of any non-essential manifold of dimension greater than two. Under some curvature assumptions, upper bounds on the length of the shortest nontrivial closed geodesic exist (see [38], [35] and [30] for general results).

The proof of M. Gromov's isosystolic inequality (1.3) rests on the two following filling radius inequalities.

TheOrem 1.3 (M. Gromov). — Let $M$ be a complete Riemannian n-manifold, then

$$
\begin{aligned}
& \operatorname{FillRad}(M) \geq \frac{1}{6} \operatorname{sys}_{1}(M) \text { if } M \text { is essential, } \\
& \operatorname{FillRad}(M) \leq c_{n} \operatorname{Vol}(M)^{1 / n} \text { for some } c_{n}>0 .
\end{aligned}
$$

In particular, the first inequality holds for all the closed surfaces except the sphere. The second inequality, more difficult to establish (though in the case of the sphere $S^{2}$ it may be obtained in a more elementary way), takes the form $\operatorname{FillRad}\left(S^{2}\right) \leq \operatorname{Area}\left(S^{2}\right)^{\frac{1}{2}}$ for the 2-sphere $S^{2}$ (see [17, p. 128]).

Thus, the inequalities (1.1) and (1.2) lead to the following corollary which improves C. Croke's result providing an alternative proof.

Corollary 1.4. - Let $M$ be a Riemannian 2-sphere, then

$$
\begin{gathered}
\operatorname{Area}(M) \geq \frac{1}{(12)^{2}} \operatorname{scg}(M)^{2}, \\
\operatorname{Area}(M) \geq \frac{1}{(20)^{2}} \bar{L}(M)^{2} .
\end{gathered}
$$


For bumpy metrics, we can replace $\bar{L}(M)$ by $L(M)$ in the above inequalities. Note that the length of a simple closed geodesic around the waist of an hourglass figure does not provide a "good" lower bound on the area as it can be made arbitrarily small while the area remains constant. These closed geodesics, which still are simple and have a null index after slight perturbations of the metric into a bumpy one, can actually be ignored to give a better bound on the area.

Note that inequality (1.6) is not optimal (C. Croke conjectures that the extremal sphere is composed of two copies of flat equilateral triangles glued together along their boundaries) and that sharp isosystolic inequalities are known only for the 2-torus, the projective plane and the Klein bottle (see [7], [33], [6] and $[36])$.

The proof of the Main Theorem rests on a minimax principle derived from Morse Theory on the space of 1 -cycles $\mathcal{Z}_{1}(M, \mathbb{Z})$ on $M$. This principle, based on F. Almgren's isomorphism $\pi_{1}\left(\mathcal{Z}_{1}\left(S^{2}, \mathbb{Z}\right),\{0\}\right) \simeq H_{2}\left(S^{2}, \mathbb{Z}\right) \simeq \mathbb{Z}$ (see [2] and Theorem 2.4 for a more general version), has been established by F. Almgren and J. Pitts using geometric measure theory and has been used by E. Calabi and J. Cao in [10]. The use of the space of 1-cycles rather than the ordinary free loop space allows us to cut and paste closed curves using several component loops. This minimax principle proceeds as follows.

Let us consider the one-parameter families $\left(z_{t}\right)_{0 \leq t \leq 1}$ of 1 -cycles on $M$ which satisfy the following conditions:

(C1) $z_{t}$ starts and ends at null-currents,

(C2) $z_{t}$ induces a nontrivial class $[z]$ in $\pi_{1}\left(\mathcal{Z}_{1}(M, \mathbb{Z}),\{0\}\right)$.

We define the minimax value

$$
L_{1}(M):=\inf _{[z] \neq 0} \sup _{0 \leq t \leq 1} \operatorname{mass}\left(z_{t}\right) .
$$

For bumpy metrics, we introduce other constructions as follows.

The previous global minimax principle extends to the nontrivial groups $\pi_{1}\left(\mathcal{Z}_{1}^{\leq \kappa_{1}}(M), \mathcal{Z}_{1}^{\leq \kappa_{0}}(M)\right)$, where $0 \leq \kappa_{0}<\kappa_{1}$ and

$$
\mathcal{Z}_{1}^{\leq \kappa}(M)=\left\{z \in \mathcal{Z}_{1}(M, \mathbb{Z}) \mid \operatorname{mass}(z) \leq \kappa\right\} .
$$

We refer to Section 4.1 for further details. The lowest positive minimax value of these local minimax processes is noted $L_{1}^{\prime}(M)$. We show that $L_{1}^{\prime}(M)$ agrees with the mass $L_{1}^{\prime \prime}(M)$ of the shortest 1-cycle of index 1. Here, the index of a 1-cycle of mass $\kappa$ is defined by

$$
\operatorname{ind}_{\mathcal{Z}_{1}}(z)=\min \left\{i \in \mathbb{N} \mid \pi_{i}\left(\mathcal{Z}_{1}^{<\kappa}(M) \cup\{z\}, \mathcal{Z}_{1}^{<\kappa}(M)\right) \text { is nontrivial }\right\} .
$$

Further, we show that $\operatorname{scg}(M) \leq L_{1}^{\prime}(M)=L_{1}^{\prime \prime}(M) \leq L_{1}(M)$.

We also introduce a new curve-shortening process which permits us to prove the following

TOME $132-2004-\mathrm{N}^{\mathrm{O}} 1$ 
TheOREM 1.5. - Let $M$ be a bumpy Riemannian 2-sphere, then

$$
\operatorname{FillRad}(M) \geq \frac{1}{20} L_{1}^{\prime \prime}(M)
$$

where $L_{1}^{\prime \prime}(M)$ is the length of the shortest 1-cycle of index 1 .

We show then that the shortest 1-cycle of index 1 for bumpy metrics is either a simple closed geodesic of index 1 or a figure-eight geodesic of null index. This immediately leads to the Main Theorem.

Contrary to $L_{1}^{\prime \prime}(M)$, the invariant $L_{1}(M)$ provides no universal lower bound on the filling radius of the 2 -sphere. More precisely, we have

THEOREM 1.6. - There exists a sequence $g_{n}$ of Riemannian metrics on $S^{2}$ which satisfies

$$
\lim _{n \rightarrow \infty} \frac{\operatorname{FillRad}\left(S^{2}, g_{n}\right)}{L_{1}\left(S^{2}, g_{n}\right)}=0 .
$$

Using techniques involved in the proof of Theorem 1.1, we also prove

TheOREM 1.7. - Let $M$ be a Riemannian 2-sphere of diameter $\operatorname{Diam}(M)$, then

$$
\operatorname{scg}(M) \leq 4 \operatorname{Diam}(M)
$$

For other simply connected manifolds, it is still unknown whether or not a similar inequality holds. Note that C. Croke already showed in [12] that $\operatorname{scg}(M) \leq 9 \operatorname{Diam}(M)$ for 2 -spheres. This inequality was then improved with the constant 5 by M. Maeda in [26].

Theorem 1.7 may also be derived from Theorem 1.1 and the sharp general filling inequality $\operatorname{FillRad}(M) \leq \frac{1}{3} \operatorname{Diam}(M)$ established by M. Katz in [23]. However, we present its short proof because it illustrates in a simple way some techniques used in this paper.

After having written the final version of this paper, the author learned that A. Nabutovsky and R. Rotman have independently established similar results. Specifically, on 2-spheres, they have obtained in [29] the same improvement for the diameter lower bound as us ( $c f$. Theorem 1.7) and a better one for the area lower bound $\left(\frac{1}{64}\right.$ instead of $\frac{1}{144}$ in $\left.(1.6)\right)$. They have also obtained in [28] a lower bound on the filling radius of any closed Riemannian manifold in terms of the mass of the shortest stationary 1-cycle.

We refer the reader to the recent survey [13] and the references therein for an account of further curvature-free geometric inequalities.

In Section 2, we study a minimax principle on the space of 1-cycles which yields a nontrivial closed geodesic on the 2 -sphere. Then, we introduce a new curve-shortening process. In Section 3, we illustrate the general use of this minimax principle and show that the length of the shortest closed geodesic on the 2 -sphere provides a lower bound on the filling radius. Section 4 is devoted to 
the proof of the filling radius estimates of the Main Theorem. The geometrical structure of the shortest 1-cycle of index 1 is described here. In Section 5, we construct a (counter)-example showing that the minimax value of the global minimax principle does not provide any lower bound on the filling radius.

The author would like to thank the referee for her/his very precise remarks and constructive criticism.

\section{Generalities and preliminaries}

In this part, we introduce the space of 1-cycles and present a minimax principle providing a nontrivial closed geodesic on the 2 -sphere. Then, we define a curve-shortening process and state its main properties.

2.1. The minimax principle on the space of 1-cycles. - Originally, G.D. Birkhoff established the existence of a nontrivial closed geodesic on the 2-sphere by using a minimax argument on the free loop space. This argument was extended in higher dimension by Fet and Lyusternik (see [25]). Since we shall need a modified version of this minimax principle, we briefly recall it.

Let $M$ be a compact Riemannian manifold such that $\pi_{k+1}(M) \neq 0$. The free loop space $\Lambda M$, formed of piecewise smooth curves $\gamma: S^{1} \rightarrow M$ parametrized proportionally to arclength, is endowed with the compact-open topology and the length functional $L$. The subspace of point curves is noted $\Lambda^{0} M$.

Recall that the closed geodesics of $M$ agree with the critical points of the energy functional $E$ on the free loop space of $M$. The (analytical) Morse index of a closed geodesic $c$ is then defined as the number (counted with multiplicity) of negative eigenvalues of $D^{2} E(c)$. It is noted $\operatorname{ind}_{\Lambda}(c)$.

A homotopically nontrivial smooth map $\varphi: S^{k+1} \rightarrow M$ induces a continuous map $\psi:\left(B^{k}, \partial B^{k}\right) \rightarrow\left(\Lambda M, \Lambda^{0} M\right)$ representing a nontrivial class in $\pi_{k}\left(\Lambda M, \Lambda^{0} M\right)$ (see [25]). Define

$$
\ell:=\inf _{\varphi \not 0} \sup _{t \in B^{k}} L\left(\psi_{t}\right) .
$$

THEOREM 2.1. - There exists a nontrivial closed geodesic of length $\ell$.

In particular, let $\left(\sigma_{t}\right)_{0 \leq t \leq 1}$ be a one-parameter family of closed curves starting and ending at point curves such that the induced map $\sigma: S^{2} \rightarrow S^{2}$ has nonzero degree. From Theorem 2.1, there exists a closed geodesic on $S^{2}$ of length $\ell$ where $\ell:=\inf _{\operatorname{deg} \sigma \neq 0} \sup _{0 \leq t \leq 1} L\left(\sigma_{t}\right)$.

REMARK 2.2. - This method sometimes yields a "short" geodesic. Actually, for 2 -spheres with nonnegative curvature, $\ell$ represents the length of the shortest nontrivial closed geodesic (see [10]). Such a result is no longer true for an arbitrary metric. In fact, the minimax value $\ell$ may be quite "long", for instance for

TOME $132-2004-\mathrm{N}^{\mathrm{O}} 1$ 
a sphere with three long spikes (see Remark 4.10 for a more precise description of this example).

Although C. Croke established a lower bound on the area in terms of the length of the shortest closed geodesic on the sphere using the Birkhoff minimax principle on $\Lambda S^{2}$, the free loop space is not very well adapted to this problem. We will rather use the space of 1-cycles.

In the remainder of this section, we introduce the space of integral 1-cycles and generalize on it the minimax principle (see [27], [32] and [10]).

We define the space $\mathcal{Z}_{1}\left(S^{2}, \mathbb{Z}\right)$ of 1 -cycles with integral coefficients over $S^{2}$ as follows

$$
\mathcal{Z}_{1}\left(S^{2}, \mathbb{Z}\right)=\left\{\sum a_{i} T_{i} \mid a_{i} \in \mathbb{Z} \text { and } T_{i} \text { is a closed rectifiable } 1 \text {-current }\right\} .
$$

It is endowed with the weak topology. Note that the weak and flat norm topologies coincide on the space of 1-cycles (see [27, §4.3]).

The space $\mathcal{Z}_{k}(M, \mathbb{Z})$ of $k$-dimensional integral cycles is defined in higher dimension in a similar manner.

The mass of a 1-current $T$ is defined by

$$
\operatorname{mass}(T)=\sup \left\{T(\omega) \mid \omega 1 \text {-form on } M \text { with } \sup _{x}|\omega(x)| \leq 1\right\} .
$$

Note that the mass functional is only semicontinuous with respect to the weak topology on the space of 1-currents. Note also that a piecewise smooth closed curve $\gamma$ with only finitely many intersection points induces by integration a 1cycle with $\operatorname{mass}(\gamma)=L(\gamma)$.

The support of a 1-form $\omega$, noted $\operatorname{Supp}(\omega)$, is defined as the closure of the set $\left\{x \in M \mid \omega(x)\right.$ is not null in $\left.T_{x} M\right\}$ in $M$.

By duality, the support of a 1-current $T$ is the smallest closed set $C$ such that

$$
\operatorname{Supp}(\omega) \cap C=\varnothing \text { implies that } T(\omega)=0 \text { for every 1-form } \omega \text { on } M .
$$

Let $T$ be a 1-current whose support decomposes into connected components $C_{i}$. The connected components of $T$ are the 1-currents $T_{i}$ defined by

$$
T_{i}(\omega)=T\left(\varphi_{i} \cdot \omega\right) \text { for every } 1 \text {-form } \omega \text { on } M,
$$

where $\varphi_{i}$ is a smooth function which equals 1 on $C_{i}$ and vanishes on $\bigcup_{j \neq i} C_{j}$. This definition does not depend on the choice of $\varphi_{i}$.

The structure of the 1-cycles is the following. A 1-cycle is indecomposable if it is induced by an oriented simple closed curve. Every 1-cycle $T$ decomposes (not necessarily in a unique way) into a sum of indecomposable 1 -cycles $T_{i}$, i.e., $T=\sum_{i \in \mathbb{N}} T_{i}$, such that $\operatorname{mass}(T)=\sum_{i \in \mathbb{N}} \operatorname{mass}\left(T_{i}\right)$ (see $[14$, p. 420]).

We describe now this structure for the local minima of the mass functional. A local minimum of the mass functional is a locally minimizing current, that is, 
a disjoint union of simple closed geodesics (see $[27, \S 8]$ ), which are of null index. The converse, given by the following lemma, is an application of Theorem 2 in $[37]$.

Lemma 2.3. - Let $M$ be a bumpy Riemannian 2-sphere. The local minima of the mass functional are strict local minima. They agree with the finite sums of disjoint simple closed geodesics of null index.

The following theorem, established by F. Almgren in [2], is a central tool in this paper. It determines the homotopy groups of the space of integral cycles.

TheOREM 2.4. - Let $M$ be a complete manifold. For every $m, k \in \mathbb{N}$ there exists a natural isomorphism

$$
\pi_{m}\left(\mathcal{Z}_{k}(M, \mathbb{Z}),\{0\}\right) \simeq H_{m+k}(M, \mathbb{Z}) .
$$

In particular, $\pi_{1}\left(\mathcal{Z}_{1}\left(S^{2}, \mathbb{Z}\right),\{0\}\right) \simeq H_{2}\left(S^{2}, \mathbb{Z}\right) \simeq \mathbb{Z}$.

For the rest of this paper, $M$ designates a Riemannian 2-sphere.

The latter isomorphism of Theorem 2.4 permits us to apply the F. AlmgrenJ. Pitts minimax principle to the 1-cycle space of the 2-sphere.

Let us consider the one-parameter families $\left(z_{t}\right)_{0 \leq t \leq 1}$ of 1 -cycles on $M$ which satisfy the following conditions:

(C1) $z_{t}$ starts and ends at null-currents,

$(\mathrm{C} 2) z_{t}$ induces a nontrivial class $[z]$ in $\pi_{1}\left(\mathcal{Z}_{1}(M, \mathbb{Z}),\{0\}\right)$.

We define the minimax value

$$
L_{1}(M):=\inf _{[z] \neq 0} \sup _{0 \leq t \leq 1} \operatorname{mass}\left(z_{t}\right)
$$

The F. Almgren-J. Pitts principle leads to the following result (see [31], [32, Theorem 4.10] and [10, Appendix] for a proof). Note that another proof is presented in Section 4.1 (see Remark 4.4).

THEOREM 2.5. - Let $M$ be a Riemannian 2-sphere. There exists a nontrivial closed geodesic on $M$ of total length $\leq L_{1}(M)$, i.e., $\operatorname{scg}(M) \leq L_{1}(M)$.

REMARK 2.6. - This minimax process can be extended in higher dimension but it is no longer clear that the obtained critical points correspond to closed geodesics (see [31] for a study of critical 1-cycles on any manifold and [32] for a generalization in term of minimal hypersurfaces).

TOME $132-2004-\mathrm{N}^{\mathrm{O}} 1$ 
2.2. Curve-shortening process. - First, we state the main properties of the curve-shortening process. The construction of the process will be presented afterwards.

For the rest of this paper, we will assume that the metric on the sphere $M$ is bumpy, that is, the closed geodesics of $M$ are non-degenerate. It is a generic condition: the space of bumpy metrics contains an open dense set of the space of metrics (see [25, p. 163]). Note that non-degenerate closed geodesics are isolated in $\Lambda M$. Therefore, there are finitely many closed geodesics of a length uniformly bounded.

The bumpy assumption is not restrictive in order to prove the inequalities of Main Theorem 1.2 and Corollary 1.4. Indeed, suppose we have established these inequalities for bumpy metrics with $L(M)$ instead of $\bar{L}(M)$ (see the introduction for the definitions). Let $g$ be an arbitrary Riemannian metric on $M$ and $g_{n}$ be a sequence of bumpy metrics which converges to $g$. The area and diameter of $\left(M, g_{n}\right)$ converge to the ones of $(M, g)$. Let $c_{n}$ be a sequence of closed geodesics on $\left(M, g_{n}\right)$ of lengths uniformly bounded. From the Palais-Smale condition (see [25, p. 26]), a subsequence of $c_{n}$, still noted $c_{n}$, converges to a closed geodesic $c$ of $(M, g)$. From the lower semi-continuity of the index, we have $\operatorname{ind}_{\Lambda}(c) \leq \operatorname{ind}_{\Lambda}\left(c_{n}\right)$ for $n$ large enough. Furthermore, if the curves $c_{n}$ are simple closed geodesics (resp. figure-eight geodesics), then the same holds for c. Therefore, $\liminf _{n \rightarrow \infty} L\left(M, g_{n}\right) \geq \bar{L}(M, g)$.

Let $\mathcal{C}$ be a finite collection of piecewise smooth curves formed either of closed curves in $\Lambda M$ or of paths and closed curves lying in a convex domain $\Omega$ homeomorphic to a disk such that the endpoints of the paths of $\mathcal{C}$ lie in the boundary of $\Omega$. A closed domain $\Omega$ is said to be convex if there is an $\varepsilon>0$ such that for all $x, y \in \Omega$ with $d(x, y)<\varepsilon$, the minimizing geodesic from $x$ to $y$ lies in $\Omega$.

The curve-shortening process depends on the collection $\mathcal{C}$ and deforms simultaneously the curves of $\mathcal{C}$. The flow of $\gamma \in \mathcal{C}$ which it induces is noted $\gamma_{t}$.

THEOREM 2.7. - Let $\mathcal{C}$ be a finite collection of curves as above. There exists a curve-shortening process defined on $\mathcal{C}$, which deforms simultaneously the curves $\gamma$ of $\mathcal{C}$ through $\gamma_{t}$ and satisfies the following properties:

(i) it leaves the endpoints of the paths fixed;

(ii) it does not increase the lengths: $\forall \gamma \in \mathcal{C}, \forall s, 0 \leq s \leq t, L\left(\gamma_{s}\right) \geq L\left(\gamma_{t}\right)$;

(iii) simple curves remain simple through the process;

(iv) non-intersecting simple curves do not intersect through the process;

(v) for every $\gamma \in \mathcal{C}$, the family $\gamma_{t}$ converges to a geodesic of null index;

(vi) the geodesics of null indices are the only fixed points of this process. 
REMARK 2.8. - The curve-shortening process depends on the whole finite collection $\mathcal{C}$ of curves. It is not defined on the whole loop space $\Lambda M$ but only for the curves of $\mathcal{C}$.

- The simple closed curves of $\mathcal{C}$ converge to local minima of the mass functional from Lemma 2.3. The closed geodesics of $\mathcal{C}$ of positive indices converge to closed geodesics of null indices like any other closed curve of $\mathcal{C}$.

- We will use all the properties of the curve-shortening process listed in Theorem 2.7, in particular in the proof of Theorem 4.8

- Other curve-evolution processes exist that satisfy some of these properties. For instance, the Birkhoff process (see [12, p. 4]) shortens curves but simple curves do not necessarily remain simple. The disk flow defined in [20] satisfies most of these properties but it may increase lengths at non-integral time, which does not yield a precise enough control on the lengths to apply minimax processes. The curvature flow defined on $\Lambda M$ satisfies these properties for embedded curves (see [16]). But the proof of this result, involving analytic techniques, has been established only for simple closed curves and not for arcs with fixed endpoints. Moreover, it is still unknown if the flow converges for non-embedded curves.

Proof of Theorem 2.7. - Without loss of generality, we can assume that the curves of $\mathcal{C}$ are polygonal (made of finitely many segments) and that none of them is a geodesic of positive index. Indeed, it is possible to deform the curves of $\mathcal{C}$ into such curves through homotopies satisfying (i)-(iv).

The curve-shortening process of $\mathcal{C}$ rests on two constructions.

Construction of the straightening process. - Let $D$ be a disk of $M$ of radius less than $\frac{1}{2} \operatorname{inj}(M)$. Given a geodesic foliation $c_{t}$ of $D$, we define a real function $h$ on $D$ by $h^{-1}(t)=c_{t}$. Let $\alpha$ be a polygonal arc and $c$ be the segment joining the endpoints of $\alpha$. Assume that $\alpha$ and $c$ form a simple closed curve and that $c$ lies in some leaf of $c_{t}$.

The goal of this straightening process is to define a homotopy $\alpha_{t}$ in $D$ between $\alpha$ and $c$ which satisfies (i)-(iii). We can slightly deform $\alpha$ through a homotopy of simple polygonal arcs, which satisfies (i)-(iii), so that the restriction $h_{\alpha}$ of $h$ to $\alpha$ satisfies:

(H1) for all $t$, the set $h_{\alpha}^{-1}(t)$ is finite or empty;

(H2) the values of $h_{\alpha}$, at its local extrema, are distinct.

Here, by definition, the local extrema are different from the endpoints of $\alpha$.

We will argue by induction on the number of local extrema of $h_{\alpha}$. The arc $\alpha$ and the segment $c$ bound a topological disk $\Delta$ in $D$. Changing the sign of $h$ and adding a constant if necessary, we can assume that $h_{\alpha}$ admits a global maximum $a^{+}$(different from the endpoints of $\alpha$ ) and that $c$ lies in $c_{0}$. Let $\alpha^{+}$ be the subarc of $\alpha$, starting and ending at local minima of $h$, such that $a^{+}$is

TOME $132-2004-\mathrm{N}^{\mathrm{O}} 1$ 


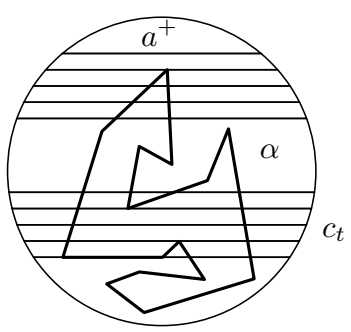

the only extremum of $h$ in the interior of $\alpha^{+}$. The pair of the endpoints of $\alpha^{+}$is noted $\partial \alpha^{+}$.

Now, we define a deformation of $\alpha^{+}$which will permit us to construct a homotopy in $\Delta$ between $\alpha$ and $c$. We deform $\alpha^{+}$by replacing the subarc of $\alpha^{+}$above $c_{s}$ with endpoints in $c_{s}$ by the segment with the same endpoints, where $s$ decreases from $h\left(a^{+}\right)$to $\max h\left(\partial \alpha^{+}\right)$. This deformation of $\alpha^{+}$, with fixed endpoints, is noted $\alpha_{t}^{+}$with $0 \leq t \leq 1$. Note that if $a^{+}$is the only extremum of $h_{\alpha}$ then $\alpha^{+}=\alpha$ and $\alpha_{t}^{+}$is a homotopy in $\Delta$ between $\alpha$ and $c$ which satisfies (i)-(iii).

Let us now define a homotopy $\alpha_{t}$ in $\Delta$ between $\alpha$ and $c$, which satisfies (i)-(iii). We argue by induction on the number of local extrema of $h_{\alpha}$.

Suppose that the homotopy $\alpha_{t}^{+}$does not pass by a local maximum of $h_{\alpha}$ different from $a^{+}$. This homotopy lies in $\Delta$ and stops for $t=1$ when $\alpha_{t}^{+}$ agrees with a segment one of whose endpoints $x_{0}$ is a local minimum of $h_{\alpha}$. Note $\left[x_{0}, x_{1}\right]$ this segment. We slightly extend the homotopy $\alpha_{t}^{+}$by deforming the segment $\left[x_{0}, x_{1}\right]$ into $\left[x_{0}, x_{t}\right]$ where $x_{t}$ moves down along $\alpha$ for $t \geq 1$. Note that it is possible from (H2). The homotopy $\alpha_{t}^{+}$so extended defines a deformation $\alpha_{t}$ in $\Delta$ from $\alpha$ to a new simple polygonal $\operatorname{arc} \beta$. The restriction $h_{\beta}$ of $h$ to $\beta$ satisfies the properties (H1)-(H2) and has fewer local extrema than $h$. We can then conclude by induction.

Suppose that the homotopy $\alpha_{t}^{+}$passes through a local maximum of $h$. Let $t_{0}$ be the greatest $t$ at which $\alpha_{t}^{+}$passes through a local maximum $x_{0}$ of $h$, different from $a^{+}$. The homotopy $\alpha_{t}^{+}$lies in $\Delta$ for $0 \leq t \leq t_{0}$. The set $\Delta \backslash \cup_{0 \leq t \leq t_{0}} \alpha_{t}^{+}$ is formed of two disjoint topological disks. One of them, noted $\Delta^{+}$, does not meet the segment $c$. Let $\alpha^{\prime}$ be the simple polygonal arc $\partial \Delta^{+} \backslash \alpha_{t_{0}}^{+}$. Let $c^{\prime}$ be the segment of $\Delta$ joining the endpoints of $\alpha^{\prime}$. By construction, the segment $c^{\prime}$ lies in some leaf of $c_{t}$ and the endpoints of $\alpha^{\prime}$ are the only intersection points with $c^{\prime}$. Moreover, the restriction $h_{\alpha^{\prime}}$ of $h$ to $\alpha^{\prime}$ satisfies the properties (H1)-(H2) and has fewer local extrema than $h_{\alpha}$. By induction, there exists a homotopy $\alpha_{t}^{\prime}$ in $\Delta$ between $\alpha^{\prime}$ and $c^{\prime}=\left[x_{0}, x_{1}\right]$. We slightly extend the homotopy $\alpha_{t}^{\prime}$ as previously, by deforming the segment $\left[x_{0}, x_{1}\right]$ into $\left[x_{0}, x_{t}\right]$ where $x_{t}$ moves up along $\alpha$. The homotopy $\alpha_{t}^{\prime}$ so extended defines a deformation $\alpha_{t}$ in $\Delta$ from $\alpha$ 
to a new arc $\beta$ such that $h_{\beta}$ has fewer local extrema than $h_{\alpha}$. By induction, the $\operatorname{arc} \beta$ straightens into $c$.

This achieves the construction of the straightening process.

Given a polygonal arc $\alpha$ and a segment $c$ with the same endpoints which form a simple closed curve, it is always possible to define a geodesic foliation $c_{t}$ such that $c$ lies in $c_{0}$ in order to apply the straightening process to $\alpha$.

We can now present the basic step of the curve-shortening process.

Let $D$ be a disk of $M$ of radius less than $\frac{1}{2} \operatorname{inj}(M)$, whose boundary $\partial D$ meets every curve of $\mathcal{C}$ transversely. If $\mathcal{C}$ is formed of arcs and closed curves lying in a convex domain $\Omega$, we replace $D$ by $D \cap \Omega$. The intersections of the curves of $\mathcal{C}$ with $D$ form a collection of subarcs $\alpha_{i}$, noted $\Gamma$. The following construction straightens every arc $\alpha_{i}$ into the segment of the same endpoints.

Construction of the curve-shortening process with respect to D. - Using the straightening process, we can deform in $D$, one at a time, the closed curves of $\Gamma$ and the loops formed of the subarcs of the curves of $\Gamma$, into point curves. Thus, we can assume that no closed curve of $\mathcal{C}$ lies in $D$ and that the $\operatorname{arcs} \alpha_{i}$ are simple. Let $c_{i}$ be the segment joining the endpoints of $\alpha_{i}$.

Let us define a homotopy in $D$ between $c_{1}$ and $\alpha_{1}$. We can slightly deform the $\operatorname{arcs} \alpha_{i}$ through a homotopy of polygonal arcs, which satisfies (i)-(iv), so that the segment $c_{1}$ cuts the new $\operatorname{arcs} \alpha_{i}$ so deformed, finitely many times, at transverse intersection points. We still denote by $\Gamma$ the new collection of $\operatorname{arcs} \alpha_{i}$ so deformed. The arcs $\alpha_{i}$ of $\Gamma$ such that $c_{i}$ has no transverse intersection point with $c_{1}$ form a subcollection $\Gamma_{1}$ of $\Gamma$. We will argue by induction on the number $N\left(c_{1}, \Gamma\right)=\sum_{\alpha_{i} \in \Gamma_{1}} \operatorname{card}\left(c_{1} \cap \alpha_{i}\right)$ of intersection points between $c_{1}$ and the $\alpha_{i}$ 's, with $\alpha_{i} \in \Gamma_{1}$. Let $\alpha$ be a subarc of some arc $\bar{\alpha}$ of $\Gamma_{1}$ such that $c_{1}$ and $\alpha$ bound a topological disk $\Delta$ of $D$. We can choose $\alpha$ and $\bar{\alpha}$ so that $\Delta$ is minimal for the inclusion. We deform in $\Delta$ the arc $\alpha$ into a segment $c$ of $c_{1}$ by applying the straightening process. This yields a homotopy between $\bar{\alpha}$ and a new arc $\bar{\alpha}^{\prime}$ such that $c$ lies in $\bar{\alpha}^{\prime}$. The new collection of arcs so obtained is noted $\Gamma^{\prime}$. Note that if no arc $\alpha_{i}$ different from $\alpha_{1}$ cuts the segment $c_{1}$, we get a homotopy between $\alpha_{1}$ and $c_{1}$. Now, we perturb $\bar{\alpha}^{\prime}$ through a homotopy of polygonal arcs by slightly deforming the segment $c$, so that $N\left(c_{1}, \Gamma^{\prime}\right)$ is less than $N\left(c_{1}, \Gamma\right)$. Such a perturbation of $\Gamma^{\prime}$ satisfying (i)-(iv) always exists. We conclude then by induction and get a homotopy between $\alpha_{1}$ and $c_{1}$. The original collection $\Gamma$ is thus deformed into a new one, noted $\bar{\Gamma}$.

We can now apply this construction to $\bar{\Gamma}$ to straighten another arc. After a finite number of iterations, all the arcs of the original collection are straightened into segments with the same endpoints. This defines the curve-shortening process with respect to $D$.

The construction of the global curve-shortening process rests on an iteration principle similar to the one of the disk flow defined in [20].

TOME $132-2004-\mathrm{N}^{\mathrm{O}} 1$ 
Let $D_{i}$ be a periodic sequence of disks of radius less than $\frac{1}{2} \operatorname{inj}(M)$. We choose the disks $D_{i}$ well-positioned relative to $\mathcal{C}$ (see $[20$, p. 26]) such that the union covers $M$. The flow $\gamma_{t}$ of $\gamma \in \mathcal{C}$ is defined for $i-1 \leq t \leq i$ as the result of performing the curve-shortening process with respect to $D$. Note that it agrees with the disk flow at integral times. The convergence of this flow to closed geodesics follows now from the proof of [20, Theorem 1.8]. We recall that the geodesics of $M$ are isolated and non-degenerate. Consider the curves $\gamma_{t}$, where $\gamma \in \mathcal{C}$, which converge to geodesics $\gamma_{\infty}$ of positive index. These curves can be deformed into $\gamma_{t}^{\prime}$, for $t$ large enough through homotopies of curves satisfying (i)-(iv), so that $L\left(\gamma_{t}^{\prime}\right)<L\left(\gamma_{\infty}\right)$. Perturbing the flow in this way, we can assume that the curves of $\mathcal{C}$ converge to geodesics of null index. This defines the curveshortening process. By construction, the properties (i)-(iv) are satisfied.

REMARK 2.9. - For a non-generic metric, the curve-shortening process may not converge to closed geodesics but oscillate between them.

The curve-shortening process satisfies the following result whose proof follows [12, Lemma 2.2] and [10, Lemma 1.1].

LEMMA 2.10. - Let $\gamma$ be a simple closed curve bounding a convex domain $\Omega$ of $M$ with $L(\gamma)<\operatorname{scg}(M)$. There exists a homotopy $\gamma_{t} \in \Lambda M$ obtained by the curve-shortening process which satisfies:

- $\gamma_{0}=\gamma$

- $\gamma_{1}$ is a point curve ;

- $\gamma_{t}$ is a simple curve of $\Omega$ bounding the convex domain

$$
\Omega_{t}:=\left\{x \in \gamma_{s} \mid t \leq s \leq 1\right\}
$$

- $L\left(\gamma_{t}\right)$ is non-increasing ;

- $\left\{\gamma_{t} \mid 0 \leq t \leq 1\right\}$ gives rise in a natural way to a map of degree \pm 1 from the two-disk $D^{2}$ onto $\Omega$.

\section{Diameter, filling radius and the shortest closed geodesic}

In this part, we first establish an upper bound on the length of the shortest closed geodesic in terms of the diameter of the 2 -sphere. Then, we show that the filling radius of the 2 -sphere is bounded from below in terms of the length of the shortest closed geodesic. Some constructions and arguments used in this part will be developed further afterwards.

3.1. Diameter and the shortest closed geodesic. — Following the ideas used in [12], we can prove

Theorem 3.1. - Let $M$ be a Riemannian 2-sphere, then

$$
\operatorname{scg}(M) \leq 4 \operatorname{Diam}(M),
$$

where $\operatorname{scg}(M)$ is the length of the shortest nontrivial closed geodesic on $M$.

BULLETIN DE LA SOCIÉTÉ MATHÉMATIQUE DE FRANCE 
REMARK 3.2. - The example of the round sphere and of the sphere composed of two copies of flat disks glued together along their boundaries shows that the optimal constant is not less than 2 .

Proof. - Without loss of generality, we will assume the metric generic. Let $x, y$ in $M$ such that $d(x, y)=\operatorname{Diam}(M)$. We can assume that $\operatorname{Diam}(M)<\frac{1}{2} \operatorname{scg}(M)$. Berger's lemma (see [11, p. 106]) asserts that for any non-zero tangent vector $v \in T_{x} M$ there exists a minimizing geodesic $\gamma$ from $x$ to $y$ such that the angle $\angle(v, \dot{\gamma}(0))$ between $v$ and $\dot{\gamma}(0)$ is not greater than $\frac{1}{2} \pi$. In the case of the sphere, it can be stated in the following more precise way (see $[12$, p. 13]).

Lemma 3.3. - Let $x, y \in M$ such that $d(x, y)=\operatorname{Diam}(M)$. There exist minimizing geodesics $\left(\gamma_{i}\right)_{1 \leq i \leq m}$ from $x$ to $y$ such that the closed curves $\gamma_{i} \cup\left(-\gamma_{i+1}\right)$ bound convex domains $\Omega_{i}$ in $M$ with $i=1, \ldots, m$.

Thus, $\mathrm{M}$ decomposes into convex domains $\Omega_{i}$ with $L\left(\partial \Omega_{i}\right)=2 \operatorname{Diam}(M)<$ $\operatorname{scg}(M)$. Lemma 2.10 asserts that there is a homotopy $c_{t}^{i} \in \Lambda M$ lying in $\Omega_{i}$ between $\alpha_{i}:=\gamma_{i} \cup\left(-\gamma_{i+1}\right)=\partial \Omega_{i}$ and a point curve $p_{i}$ which satisfies $L\left(c_{t}^{i}\right) \leq L\left(\partial \Omega_{i}\right)$.

We define a one-parameter family of 1-cycles on $M$ as follows. We start from the sum of the points $p_{1}, \ldots, p_{m-1}$. We deform $p_{1}$ into $\alpha_{1}$ via the homotopy $c_{t}^{1}$, then we deform $p_{2}$ into $\alpha_{2}$ via the homotopy $c_{t}^{2}$. At this stage, the 1-cycle obtained is the sum $\alpha_{1}+\alpha_{2}=\gamma_{1}-\gamma_{3}$ (as 1-cycles). Then, we deform $p_{3}$ to $\alpha_{3}$ through the homotopy $c_{t}^{3}$. We get the 1 -cycle $\alpha_{1}+\alpha_{2}+\alpha_{3}=\gamma_{1}-\gamma_{4}$. We repeat this process so on, deforming $p_{i}$ to $\alpha_{i}$ through the homotopy $c_{t}^{i}$ one at a time. We obtain a homotopy from the null-current to $\gamma_{1}-\gamma_{m}$. Using the homotopy $c_{t}^{m}$, we contract this latter curve into the null-current.

Since the homotopies $c_{t}^{i}$ give rise to maps of degree one onto each domain $\Omega_{i}$ of the decomposition of $M$, the one-parameter family of 1-cycles $z_{t}$ defined above satisfies $(\mathrm{C} 1)-(\mathrm{C} 2)$. Moreover, the mass of each of these 1-cycles is bounded from above by $4 \operatorname{Diam}(M)$. Therefore, from the minimax principle of Theorem 2.5, there exists a nontrivial closed geodesic of length $\leq 4 \operatorname{Diam}(M)$, i.e., $\operatorname{scg}(M) \leq 4 \operatorname{Diam}(M)$.

3.2. Filling radius and the shortest closed geodesic. - In what follows, the sphere $M$ is considered isometrically embedded, as metric space, in $L^{\infty}(M)$ (see the definition of the filling radius in the introduction).

Let $L_{0}(M)$ be the length of the shortest nontrivial simple closed geodesic on $M$ of null index. Equivalently, $L_{0}(M)$ is the smallest length of the nontrivial local minima of the mass functional (see Lemma 2.3). In particular, every simple closed curve of length less than $L_{0}(M)$ converges to a point curve through the curve-shortening process. We refer to (2.1) for the definition of $L_{1}(M)$.

TOME $132-2004-\mathrm{N}^{\mathrm{O}} 1$ 
TheOREM 3.4. - Let $M$ be a Riemannian 2-sphere, then

$$
\operatorname{FillRad}(M) \geq \min \left\{\frac{1}{6} L_{0}(M), \frac{1}{12} L_{1}(M)\right\} .
$$

In particular, FillRad $(M) \geq \frac{1}{12} \operatorname{scg}(M)$, where $\operatorname{scg}(M)$ is the length of the shortest nontrivial closed geodesic on $M$.

REMARK 3.5. - The constant $\frac{1}{12}$ in the last inequality of Theorem 3.4 should be compared with the constant $\frac{1}{6}$ in the sharp filling inequality (1.4), which is reached for instance by the flat tori.

Proof. - We follow the arguments of [17, Lemma 1.2.B]. By definition, the fundamental class $[M]$ of $M$ vanishes in $U_{\delta}(M) \subset L^{\infty}(M)$ where $\delta>\operatorname{Fill} \operatorname{Rad}(M)$. Therefore, there exists a 3-dimensional chain $c$ in $U_{\delta}(M)$, with coefficients in $\mathbb{Z}$, which fills $M$, i.e., whose boundary $\partial c$ contained in $M$ represents $[M]$. Using a piecewise linear approximation of $c$, we construct a 3-dimensional polyhedron $P$ in $U_{\delta}(M)$ representing $c$ and containing a sub-complex $Q \subset M$ which represents $\partial c$, that is, $[M]$. In this case, the induced natural map $i_{*}: H_{2}(M) \rightarrow H_{2}\left(U_{\delta}(M)\right)$ is null.

Let $P$ be a 3 -dimensional polyhedron in $U_{\delta}(M)$ containing $M$ as a subpolyhedron, i.e., $M \subset P \subset U_{\delta}(M) \subset L^{\infty}(M)$. Fix $\delta>0$. We show that if $\delta<\min \left\{\frac{1}{6} L_{0}(M), \frac{1}{12} L_{1}(M)\right\}$, the inclusion $M \hookrightarrow P$ admits a retraction. Thus $M$ does not bound in $U_{\delta}(M)$, hence the inequality.

Subdividing $P$ if necessary, we can assume that the diameter of all simplices in $P$ is less than $\varepsilon>0$ with $\varepsilon<\min \left\{\frac{1}{3} L_{0}(M), \frac{1}{6} L_{1}(M)\right\}-2 \delta$ and $\varepsilon<\operatorname{conv}(M)$, where $\operatorname{conv}(M)$ is the convexity radius of $M$. Using the Birkhoff process with fixed endpoints if necessary, we can also assume that the edges of $P$ lying in $M$ are minimizing segments. We are going to construct successively on the skeleton of $P$ a retraction $r: P \rightarrow M$.

We define $r: P^{0} \rightarrow M$ on the 0 -skeleton of $P$ by sending each vertex $p_{i}$ of $P$ to a nearest point $v_{i}$ of $M$, as we wish. The vertices of $M$ are thus fixed.

Let us extend this map to the 1-skeleton $P^{1}$ of $P$. Since $i: M \hookrightarrow U_{\delta}(M)$ preserves the distances, we have for every pair $p_{i}, p_{j}$ of adjacent vertices of $P$

$$
\begin{aligned}
d_{M}\left(v_{i}, v_{j}\right) & =d_{L^{\infty}(M)}\left(v_{i}, v_{j}\right) \\
& \leq d_{L^{\infty}(M)}\left(v_{i}, p_{i}\right)+d_{L^{\infty}(M)}\left(p_{i}, p_{j}\right)+d_{L^{\infty}(M)}\left(p_{j}, v_{j}\right) \\
& \leq 2 \delta+\varepsilon=: \rho<\min \left\{\frac{1}{3} L_{0}(M), \frac{1}{6} L_{1}(M)\right\}
\end{aligned}
$$

where $v_{i}$ and $v_{j}$ are the images by $r$ of $p_{i}$ and $p_{j}$. We map $\left[p_{i}, p_{j}\right]$ to a minimizing segment $\left[v_{i}, v_{j}\right] \subset M$ joining $v_{i}$ to $v_{j}$. This defines an extension $P^{1} \rightarrow M$ which leaves the edges of $M$ fixed.

Let us now extend this map to $P^{2}$. Let $\Delta^{2}$ be a 2-simplex of $P$ which lies in $M$. We extend $r$ by the identity on $\Delta^{2}$. Since the edges of $\Delta^{2}$ are 
minimizing segments and $\operatorname{Diam}\left(\Delta^{2}\right) \leq \operatorname{conv}(M), \Delta^{2}$ is convex and the curveshortening process contracts $\partial \Delta^{2}$ into a point through a homotopy of free loops lying in $\Delta^{2}$ (see Lemma 2.10). Let $\Delta^{2}$ be a 2-simplex of $P$ which does not lie in $M$. Since the boundary $\partial \Delta^{2}$ maps onto a geodesic triangle $T$ of perimeter less than $3 \rho<L_{0}(M)$, the curve-shortening process defines a map from the disk $D \simeq \Delta^{2}$ to $M$ which sends $\partial D \simeq \partial \Delta^{2}$ onto $T$. This construction yields a map $r: P^{2} \rightarrow M$ whose restriction to $M$ is the identity.

We now want to extend it to $P^{3}$. This is possible if the restriction $\varphi: \partial \Delta \rightarrow M$ of $r: P^{2} \rightarrow M$ to the boundary $\partial \Delta$ of each 3 -simplex $\Delta$ of $P$ is of null degree. We recall that $\partial \Delta$ is homeomorphic to a sphere. The curve-shortening process used in the construction of $r: P^{2} \rightarrow M$ provides homotopies $c_{t}^{i}$ defined on each face $\Delta_{i}$ of $\Delta$ between $\partial \Delta_{i}$ and point curves $p_{i}$ such that $L\left(\varphi\left(c_{t}^{i}\right)\right) \leq L\left(\partial \Delta_{i}\right)$.

We define a one-parameter family of 1-cycles on $M$ putting together the homotopies $c_{t}^{1}+c_{t}^{2}$ and $c_{t}^{3}+c_{t}^{4}$. The construction is analogous to the one carried out in the proof of Theorem 3.1. We start from the sum of $p_{1}$ and $p_{2}$. We deform it through the sum of the homotopies $c_{t}^{1}$ and $c_{t}^{2}$ into $\partial \Delta_{1}+\partial \Delta_{2}$ with $\partial \Delta_{1}+\partial \Delta_{2}=-\partial \Delta_{3}-\partial \Delta_{4}$ as 1-cycles. Then, we deform $-\partial \Delta_{3}-\partial \Delta_{4}$ into $-p_{3}-p_{4}$ using the homotopies $c_{t}^{3}$ and $c_{t}^{4}$.

The one-parameter family $z_{t}$ of 1-cycles on $\partial \Delta$ so defined starts and ends at null-currents. Since the homotopies $c_{t}^{i}$ give rise to maps of degree one on each face $\Delta_{i}$ of $\partial \Delta$, it represents a generator of $\pi_{1}\left(\mathcal{Z}_{1}(\partial \Delta),\{0\}\right) \simeq \pi_{2}(\partial \Delta) \simeq \mathbb{Z}$. Moreover, $\operatorname{mass}\left(\varphi\left(z_{t}\right)\right) \leq 6 \rho<L_{1}(M)$. Therefore, by definition of $L_{1}(M)$, the family $\varphi\left(z_{t}\right)$ represents a null-class in $\pi_{1}\left(\mathcal{Z}_{1}(M),\{0\}\right)$. So,

$$
\varphi_{*}: \pi_{1}\left(\mathcal{Z}_{1}(\partial \Delta),\{0\}\right) \simeq \pi_{2}(\partial \Delta) \longrightarrow \pi_{1}\left(\mathcal{Z}_{1}(M),\{0\}\right) \simeq \pi_{2}(M)
$$

is trivial. That is the degree of $\varphi$ is null.

In conclusion, $\varphi: \partial \Delta \rightarrow M$ can be extended to $\Delta$ for each 3 -simplex $\Delta$ of $P^{3}$. Therefore, the inclusion $M \hookrightarrow P$ admits a retraction.

\section{Filling radius and the minimal 1-cycle of index 1}

We will first introduce a local minima process on the 1-cycle space and define the index of the 1-cycles. Then, we will describe the structure of the shortest 1-cycle of index 1 on the 2-sphere. Finally, we will show that the length of the latter provides a lower bound on the filling radius, extending the results of the previous section.

4.1. Indices of the 1-cycles. - In this section, we will use the language of the varifolds to state some results due to F. Almgren and J. Pitts. However, in order to be consistant with the use of the 1-cycles, we will restate these results in the language of 1-cycles.

TOME $132-2004-\mathrm{N}^{\mathrm{O}} 1$ 
The varifolds considered in this section correspond to unions of closed curves counted with multiplicity. Since we will not really make use of the varifolds in the proofs of the results which follow, the latter will not be defined here. Instead, we refer to [32, p. 60-62] for the definition of the 1-dimensional integral varifolds and to $[32$, p. 74] for the one of stationary varifolds.

The global minimax process on the 1-cycle space described by $(\mathrm{C} 1)-(\mathrm{C} 2)$ in Section 2.1 admits a local version presented below: given $\kappa>0$, let

$$
\mathcal{Z}_{1}^{\leq \kappa}(M)=\left\{z \in \mathcal{Z}_{1}(M, \mathbb{Z}) \mid \operatorname{mass}(z) \leq \kappa\right\} .
$$

Similarly, we define $\mathcal{Z}_{1}^{<\kappa}(M), \Lambda^{\leq \kappa}(M)$ and $\Lambda^{<\kappa}(M)$. Fix $0 \leq \kappa_{0}<\kappa_{1}$.

Let us consider the one-parameter families $\left(z_{t}\right)_{0 \leq t \leq 1}$ of 1 -cycles on $M$ which satisfy the following conditions:

$\left(\mathrm{C}^{\prime} 1\right) z_{0}, z_{1}$ lie in $\mathcal{Z}_{1}^{\leq \kappa_{0}}(M)$;

$\left(\mathrm{C}^{\prime} 2\right) z_{t}$ induces a nontrivial class $[z]$ in $\pi_{1}\left(\mathcal{Z}_{1}^{\leq \kappa_{1}}(M), \mathcal{Z}_{1}^{\leq \kappa_{0}}(M)\right)$.

We define the minimax value

$$
L\left(\kappa_{0}, \kappa_{1}\right):=\inf _{[z] \neq 0} \sup _{0 \leq t \leq 1} \operatorname{mass}\left(z_{t}\right) .
$$

This local minimax process gives rise to a stationary 1-dimensional integral varifold of total length $L\left(\kappa_{0}, \kappa_{1}\right)$ corresponding to a union of closed curves counted with multiplicity (see [31, p. 468] and [32, Theorem 4.10]). Strictly speaking, this result is stated for the global minimax process $(\mathrm{C} 1)-(\mathrm{C} 2)$, i.e., when $\kappa=0$, but it also holds with the local minimax process $\left(\mathrm{C}^{\prime} 1\right)-\left(\mathrm{C}^{\prime} 2\right)$. Thus, there exists a 1-cycle $z$ of mass $L\left(\kappa_{0}, \kappa_{1}\right)$ which induces a stationary varifold. Note that since the varifold is stationary, it decomposes $M$ into convex domains. Therefore, $L\left(\kappa_{0}, \kappa_{1}\right) \geq \operatorname{conv}(M)$ where $\operatorname{conv}(M)$ is the convexity radius of $M$.

We also define

$$
\begin{aligned}
& L_{1}^{\prime}(M)=\inf \left\{L\left(\kappa_{0}, \kappa_{1}\right) \mid 0 \leq \kappa_{0}<\kappa_{1}\right. \text { such that the } \\
& \text { conditions } \left.\left(\mathrm{C}^{\prime} 1\right)-\left(\mathrm{C}^{\prime} 2\right) \text { apply }\right\} .
\end{aligned}
$$

Note that $L_{1}^{\prime}(M)$ is positive. We have $0<L_{1}^{\prime}(M) \leq L_{1}(M)$ from the definition of $L_{1}(M)($ see $(2.1))$.

REMARK 4.1. - From the structure previously described of the critical points of the local minimax processes and the compactness theorem for stationary 1-dimensional integral varifolds (see [32, p. 77]), there exists a 1-cycle $z$ of mass $L_{1}^{\prime}(M)$ which induces a stationary varifold.

We define the index of the 1-cycles by analogy with the index of the closed geodesics. Recall that the metric is supposed bumpy. Using classical Morse theory on a finite dimensional approximation of $\Lambda M$ (see also [25, §2.4]), we see that the index of a closed geodesic $\gamma$ of length $\kappa$ agrees with

$$
\operatorname{ind}_{\Lambda}(\gamma)=\min \left\{i \in \mathbb{N} \mid \pi_{i}\left(\Lambda^{<\kappa}(M) \cup\{\gamma\}, \Lambda^{<\kappa}(M)\right) \text { is nontrivial }\right\} .
$$


The index of a 1-cycle of mass $\kappa$ is similarly defined by

$$
\operatorname{ind}_{\mathcal{Z}_{1}}(z)=\min \left\{i \in \mathbb{N} \mid \pi_{i}\left(\mathcal{Z}_{1}^{<\kappa}(M) \cup\{z\}, \mathcal{Z}_{1}^{<\kappa}(M)\right) \text { is nontrivial }\right\} .
$$

In particular, the 1-cycles of null indices agree with the local minima of the mass functional described in Lemma 2.3. Note that the varifolds induced by 1-cycles of finite indices are stationary.

We define

$$
L_{1}^{\prime \prime}(M)=\inf \{\operatorname{mass}(z) \mid z \text { is a 1-cycle of index } 1\} .
$$

The following useful claim, which is a version of the mountain pass lemma, comes from the definition of $L_{1}^{\prime}(M)$.

Claim 4.2. - Let $\left(z_{t}\right)_{0 \leq t \leq 1}$ be a homotopy of 1-cycles joining two local minima of the mass functional. If $\sup _{0 \leq t \leq 1} \operatorname{mass}\left(z_{t}\right)<L_{1}^{\prime}(M)$, then the two local minima of the mass functional agree.

Proof. - Define $\kappa_{0}=\max \left\{\operatorname{mass}\left(z_{0}\right), \operatorname{mass}\left(z_{1}\right)\right\}$ and $\kappa_{1}=\sup _{0 \leq t \leq 1} \operatorname{mass}\left(z_{t}\right)$. Since $\kappa_{1}<L_{1}^{\prime}(M)$, the group $\pi_{1}\left(\mathcal{Z}_{1}^{\kappa_{1}}(M), \mathcal{Z}_{1}^{\kappa_{0}}(M)\right)$ is trivial. Therefore, the family $z_{t}$ is homotopic to another family of 1-cycles $z_{t}^{\prime}$ with the same endpoints such that $\operatorname{mass}\left(z_{t}^{\prime}\right) \leq \kappa_{0}$. Since the endpoints $z_{0}$ and $z_{1}$ of $z_{t}^{\prime}$ are strict local minima of the mass functional (see Lemma 2.3), the family $z_{t}^{\prime}$ is constant. In particular, $z_{0}$ and $z_{1}$ agree.

The structure of the 1-cycles critical for the mass functional is described by the following

THEOREM 4.3. - Let $M$ be a bumpy Riemannian 2-sphere. We have $L_{1}^{\prime}(M)=L_{1}^{\prime \prime}(M)$. Moreover, there exists a (shortest) 1-cycle of index 1 of mass $L_{1}^{\prime \prime}(M)$. The shortest 1 -cycle of index 1 agrees with the shortest curve among

- the simple closed geodesics of indices one as geodesic;

- the figure-eight geodesics of null indices as geodesic.

REMARK 4.4. - - Both simple closed geodesics of index 1 and figure-eight geodesics of null index can occur as the shortest 1-cycles of index 1. Examples are presented in Remark 4.10.

- Since $L_{1}^{\prime}(M) \leq L_{1}(M)$, Theorem 4.3 admits Theorem 2.5 as corollary.

We will need the following result.

LEMMA 4.5. - (i) Every simple closed geodesic of positive index $\gamma$ satisfies $L(\gamma) \geq L_{1}^{\prime}(M)$. Furthermore, the inequality is strict if $\operatorname{ind}_{\Lambda}(\gamma)>1$.

(ii) Every figure-eight geodesic $\gamma$ satisfies $L(\gamma) \geq L_{1}^{\prime}(M)$. Furthermore, the inequality is strict if $\operatorname{ind}_{\Lambda}(\gamma)>0$.

TOME $132-2004-\mathrm{N}^{\mathrm{O}} 1$ 
Proof. - Let us show first the point (i). Let $\gamma$ be a simple closed geodesic of positive index. The curve $\gamma$ decomposes $M$ into two disks $D^{+}$and $D^{-}$.

We are going to define a homotopy $\gamma_{t} \in \Lambda M$ with $-1 \leq t \leq 1$, joining two simple closed geodesics of null indices, such that $\gamma_{0}=\gamma, \gamma_{t}$ lies in $D^{+}$for $t \geq 0$, $\gamma_{t}$ lies in $D^{-}$for $t \leq 0$ and $L\left(\gamma_{t}\right)<L(\gamma)$ for $t \neq 0$. But first, let us show how this yields the point (i) of the lemma.

If $\gamma_{1}$ and $\gamma_{-1}$ are not both trivial, the endpoints of the family of 1-cycles $z_{t}$ induced by $\gamma_{t}$ are different. Therefore, $L(\gamma)=\sup _{0<t<1} L\left(\gamma_{t}\right) \geq L_{1}^{\prime}(M)$ from Claim 4.2. If $\gamma_{1}$ and $\gamma_{-1}$ are not both trivial, the homotopies $\left(\gamma_{t}\right)_{0<t<1}$ and $\left(\gamma_{t}\right)_{-1 \leq t \leq 0}$ give rise to maps of degree one onto $D^{+}$and $D^{-}$. Thus, the family $z_{t}$ satisfies the conditions $(\mathrm{C} 1)-(\mathrm{C} 2)$. Therefore,

$$
L(\gamma)=\sup _{0 \leq t \leq 1} L\left(\gamma_{t}\right) \geq L_{1}(M) \geq L_{1}^{\prime}(M)
$$

In both cases, the homotopy $\gamma_{t}$ starting and ending at local minima of the mass functional satisfies (C'.1-2) for some $0 \leq \kappa_{0}<\kappa_{1}$. If further $\operatorname{ind}_{\Lambda}(\gamma)>1$, then the family $\gamma_{t}$ is homotopic in $\Lambda^{\kappa_{1}} M$ to another family $\gamma_{t}^{\prime}$ with the same endpoints such that $\sup _{0 \leq t \leq 1} L\left(\gamma_{t}^{\prime}\right)<L(\gamma)$. Therefore, $L(\gamma)>L_{1}^{\prime}(M)$.

Now, let us define the homotopy $\gamma_{t}$. The subspace of the space of piecewise smooth normal vectorfields on $\gamma$ spanned by the eigenvectors of $D^{2} E(\gamma)$ corresponding to negative eigenvalues is nontrivial. Let $V$ be an eigenvector corresponding to the smallest eigenvalue such that $\sup _{x \in \gamma}|V(x)|=1$. Let $\gamma_{t} \in \Lambda M$ be a homotopy of simple closed curves with $-1 \leq t \leq 1$ such that $\gamma_{0}=\gamma$, $\mathrm{d} \gamma_{t} / \mathrm{d} t_{\mid t=0}=V$ and $L\left(\gamma_{t}\right)<L(\gamma)$ for $t \neq 0$. We can also assume that $\gamma_{t}(x)$ is fixed at the points $x$ where $V(x)$ vanishes.

If the vectors $V(x)$ point toward $D^{+}$for every $x \in \gamma$, then $\gamma_{t}$ lies in $D^{+}$for $t \geq 0$ and $\gamma_{t}$ lies in $D^{-}$for $t \leq 0$. We deform $\gamma_{1}$ in $D^{+}$and $\gamma_{-1}$ in $D^{-}$into simple closed geodesics of null indices through the curve-shortening process. This yields the desired homotopy. The same holds true if the vectors $V(x)$ point toward $D^{-}$for every $x \in \gamma$.

Thus, we can suppose that there are two subarcs $\gamma_{t}^{+}$and $\gamma_{t}^{-}$of $\gamma_{t}$ with fixed endpoints such that $\gamma_{t}^{+}$lies in $D^{+}$and $\gamma_{t}^{-}$lies in $D^{-}$.

We have $\mathrm{d} L\left(\gamma_{t}^{+}\right) / \mathrm{d} t_{\mid t=0} \leq 0$. Otherwise, the normal vectorfield on $\gamma$ defined by $\bar{V}=V$ off $\gamma_{0}^{+}$and $\bar{V}=-V$ on $\gamma_{0}^{+}$would satisfy $\sup _{x \in \gamma}|\bar{V}(x)|=1$ and $D^{2} E_{\mid \gamma}(\bar{V}, \bar{V})<D^{2} E_{\mid \gamma}(V, V)$. Hence a contradiction with the definition of $V$.

We can now define a homotopy $\bar{\gamma}_{t}$ starting from $\gamma$, such that $\bar{\gamma}_{t}$ lies in $D^{+}$ for $t \geq 0$ and $L\left(\bar{\gamma}_{t}\right)<L(\gamma)$ for $t \neq 0$, by deforming $\gamma_{0}^{+}$through $\gamma_{t}^{+}$and smoothing out at the endpoints of $\gamma_{t}^{+}$. Then, we deform $\bar{\gamma}_{1}$ in $D^{+}$into a simple closed geodesic of null index through the curve-shortening process. We perform the same construction in $D^{-}$with $\gamma_{t}^{-}$and put together the homotopies in order to obtain the desired homotopy. 
Let us now show the point (ii). We will argue as previously. Let $\gamma$ be a figureeight geodesic. The curve $\gamma$ decomposes into two geodesic loops $\gamma^{+}$and $\gamma^{-}$, which bound convex domains $\Omega^{+}$and $\Omega^{-}$. These loops form a closed curve $c=\gamma^{+} \cup\left(-\gamma^{-}\right)$, which bounds the convex domain $\Omega:=M \backslash\left(\Omega^{+} \cup \Omega^{-}\right)$. The curves $c, \gamma^{+}$and $\gamma^{-}$, bounding the convex domains $\Omega, \Omega^{+}$and $\Omega^{-}$, converge respectively in $\Omega, \Omega^{+}$and $\Omega^{-}$to local minima $\alpha, \alpha^{+}$and $\alpha^{-}$of the mass functional through homotopies $h_{t}, h_{t}^{+}$and $h_{t}^{-}$given by the curve-shortening process. If the curve $\alpha$ is trivial, the homotopy $h_{t}$ gives rise to a map of degree one onto $\Omega$ (see Lemma 2.10). The same goes for $\gamma^{+}$and $\gamma^{-}$. Put together, the homotopies of 1-cycles $h_{t}$ and $h_{t}^{+}-h_{t}^{-}$form a one-parameter family of 1cycles $z_{t}$ with endpoints $\alpha$ and $\alpha^{+}-\alpha^{-}$. As previously, this family $z_{t}$, starting and ending at local minima of the mass functional, satisfies $\left(\mathrm{C}^{\prime} 1\right)-\left(\mathrm{C}^{\prime} 2\right)$ for some $0 \leq \kappa_{0}<\kappa_{1}$. Therefore,

$$
L(\gamma)=\sup _{0 \leq t \leq 1} \operatorname{mass}\left(z_{t}\right) \geq L_{1}^{\prime}(M) .
$$

Suppose now that the index of $\gamma$ is positive. The geodesic $\gamma$ can be slightly deformed through a path $\left(\gamma_{t}\right)_{0 \leq t \leq 1} \in \Lambda M$ of figure-eight curves with $\gamma_{0}=\gamma$ and $L\left(\gamma_{t}\right)<L(\gamma)$ for $t \neq 0$. The curves of the path $\gamma_{t}$ decompose into loops $\gamma_{t}^{+}$ and $\gamma_{t}^{-}$. These latter give rise to a path $c_{t}=\gamma_{t}^{+} \cup\left(-\gamma_{t}^{-}\right) \in \Lambda M \operatorname{starting}$ from $c$.

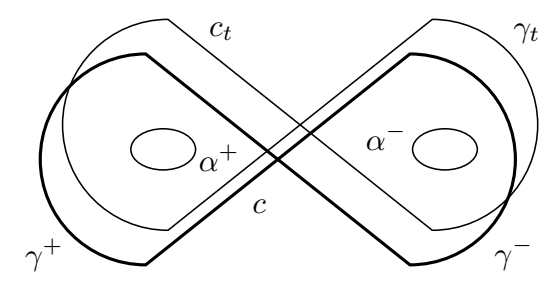

Let us now define another family of 1-cycles homotopic to $z_{t}$ with the same endpoints. Put together, the homotopies $c_{t}$ and $h_{t}$ define a homotopy in $\Lambda M$ between $c_{1}$ and $\alpha$. The length of the curves of this homotopy is $<L(\gamma)$, except for $c$. Since $c$ is not a geodesic, this homotopy deforms in $\Lambda M$ into a path $c_{1, t}$ with the same endpoints $c_{1}$ and $\alpha$ such that $L\left(c_{1, t}\right)<L(\gamma)$ for every $t$. Similarly, we define a homotopy $\gamma_{1, t}^{+}\left(\operatorname{resp} . \gamma_{1, t}^{-}\right)$between $\gamma_{1}^{+}$and $\alpha^{+}$ (resp. $\gamma_{1}^{-}$and $\alpha^{-}$) using $\gamma_{t}^{+}$and $h_{t}^{+}$(resp. $\gamma_{t}^{-}$and $h_{t}^{-}$). Put together, the homotopies of 1-cycles $c_{1, t}$ and $\gamma_{1, t}^{+}-\gamma_{1, t}^{-}$form a one-parameter family of 1 cycles $z_{1, t}$ homotopic to $z_{t}$ with the same endpoints $\alpha$ and $\alpha^{+}-\alpha^{-}$. Thus, the family $z_{1, t}$ also satisfies (C'.1-2) for some $0 \leq \kappa_{0}<\kappa_{1}$. By construction, $\sup _{0 \leq t \leq 1} \operatorname{mass}\left(z_{1, t}\right)<L(\gamma)$. Hence the conclusion.

We will also need the following result.

LEMMA 4.6. - The connected 1-cycles of mass $\leq L_{1}^{\prime}(M)$ which induce stationary varifolds are either simple closed geodesics or figure-eight geodesics.

TOME $132-2004-\mathrm{N}^{\mathrm{O}} 1$ 
Proof. - Let $\gamma$ be a connected 1-cycle of mass $\leq L_{1}^{\prime}(M)$ which induces a stationary varifold, in particular $\operatorname{mass}(\gamma) \leq L_{1}^{\prime}(M)$. Assume that $\gamma$ is neither a simple closed curve nor a figure-eight curve. In which case, $\gamma$ would be geodesic.

There exist $k$ domains $D_{i}$ such that $M \backslash \gamma=\amalg_{i \in I} D_{i}$ where $I=\{0, \ldots, k-1\}$. Since the varifold induced by $\gamma$ is stationary and is not a simple closed geodesic, the domains $D_{i}$ are convex and their boundaries $\gamma_{i}$ are not geodesics. Thus, the boundaries $\gamma_{i}$ of the convex domains $D_{i}$ converge in $D_{i}$ to local minima $\alpha_{i}$ of the mass functional through homotopies $\gamma_{i, t}$ given by the curve-shortening process. Given $x_{0} \in D_{0}$, the index of $D_{i}$ is defined as the winding number of $\gamma$ around any point of $D_{i}$ in the plane $M \backslash x_{0}$. The set $I$ decomposes into the disjoint union of $I^{+}$and $I^{-}$where the index of $D_{i}$ is even if $i \in I^{+}$and odd if $i \in I^{-}$. We have

$$
\sum_{i \in I^{+}} \gamma_{i}=-\sum_{i \in I^{-}} \gamma_{i}
$$

where the orientation of $\gamma_{i}$ is given by the one on $D_{i}$. Put together, the homotopies of 1-cycles $\sum_{i \in I^{+}} \gamma_{i, t}$ and $-\sum_{i \in I^{-}} \gamma_{i, t}$ form a one-parameter family of 1-cycles $z_{t}$ between $\sum_{i \in I^{+}} \alpha_{i}$ and $-\sum_{i \in I^{-}} \alpha_{i}$ with $\operatorname{mass}\left(z_{t}\right) \leq \operatorname{mass}(\gamma)$. Since the $\alpha_{i}$ 's are disjoint, the family $z_{t}$ starts and ends at local minima of the mass functional (see Lemma 2.3). Moreover, $z_{0}$ and $z_{1}$ are either different or both trivial. As previously, the family $z_{t}$ satisfies $\left(\mathrm{C}^{\prime} 1\right)-\left(\mathrm{C}^{\prime} 2\right)$ for some $0 \leq \kappa_{0}<\kappa_{1}$. Therefore, $\operatorname{mass}(\gamma) \geq \sup _{0 \leq t \leq 1} \operatorname{mass}\left(z_{t}\right) \geq L_{1}^{\prime}(M)$.

We are going to modify $z_{t}$ into a homotopic family $z_{t}^{\prime}$ with the same endpoints which satisfies $\sup _{0 \leq t \leq 1} \operatorname{mass}\left(z_{t}^{\prime}\right)<\operatorname{mass}(\gamma)$. This implies $\operatorname{mass}(\gamma)>L_{1}^{\prime}(M)$ and leads to a contradiction since $\operatorname{mass}(\gamma) \leq L_{1}^{\prime}(M)$.

Suppose that the indices of two adjacent domains $D_{i}$ have the same parity, even for instance. Renumbering the $D_{i}$ 's if necessary, we can assume that these two domains are $D_{0}$ and $D_{2}$.

Let $c$ be a subarc of $\gamma_{0}$ formed of two segments one of whose agrees with a common side of $D_{0}$ and $D_{2}$. The arc $c$ is a piecewise geodesic broken at some point $p$. We shorten $c$ in $D_{0}$ at the neighborhood of $p$ while keeping its endpoints fixed by smoothing it out at $p$. This yields a length-decreasing homotopy $c_{t}$ from $c$ to some arc $c^{\prime}$.

Let us consider the homotopy of 1-cycles $\gamma_{0, t}+\sum_{i \in I^{+} \backslash\{0\}} \alpha_{i}$ starting from $\sum_{i \in I^{+}} \alpha_{i}$ and joining $\gamma_{0}+\sum_{i \in I^{+} \backslash\{0\}} \alpha_{i}$. We extend it by deforming first the curve $\alpha_{2}$ into $\gamma_{2}$ through $\gamma_{2, t}$. We get a 1-cycle which agrees with

$$
\gamma_{0}^{\prime}+\gamma_{2}+\sum_{i \in I^{+} \backslash\{0,2\}} \alpha_{i},
$$

where $\gamma_{0}^{\prime}=\left(\gamma_{0} \backslash c\right) \cup c^{\prime}$. Note that $\gamma_{0}^{\prime}$ and $\gamma_{2}$ have a nontrivial segment in common with opposite orientations. This segment vanishes out in the sum of 1-cycles $\gamma_{0}^{\prime}+\gamma_{2}$. Then, we deform $c^{\prime}$ into $c$ through $c_{t}$ and $\alpha_{i}$ into $\gamma_{i}$ through $\gamma_{i, t}$ 
to obtain

$$
\sum_{i \in I^{+}} \gamma_{i}=-\sum_{i \in I^{-}} \gamma_{i}
$$

Finally, we deform the 1-cycle so obtained into $-\sum_{i \in I^{-}} \alpha_{i}$ through $-\sum_{i \in I^{-}} \gamma_{i, t}$. As a result, we get a one-parameter family of 1-cycles $z_{t}^{\prime}$ homotopic to $z$ with the same endpoints. By construction, there exists $\varepsilon>0$ such that $\operatorname{mass}\left(z_{t}\right)+\varepsilon \leq \operatorname{mass}(\gamma) \leq L_{1}^{\prime}(M)$. Hence the result.

We can now assume that the indices of adjacent domains $D_{i}$ have different parities. From this assumption and since $\gamma$ is not simple, there exists $p \in \gamma$ such that the (finite) set $\mathcal{V} \subset U_{p} M$ of unit vectors based at $p$ tangent to $\gamma$ has at least four vectors. Every pair of adjacent vectors of $\mathcal{V}$ spans a domain $D_{i}$ for some $i \in I$. There exist two different domains in $\left\{D_{i} \mid i \in I\right\}$ spanned by two pairs, $\{u, v\}$ and $\left\{u^{\prime}, v^{\prime}\right\}$, of vectors of $\mathcal{V}$ such that $u, v, u^{\prime}$ and $v^{\prime}$ are consecutive for the cyclic order on $U_{p} M$. Renumbering the $D_{i}$ 's if necessary, we can assume that the pairs $\{u, v\},\left\{v, u^{\prime}\right\}$ and $\left\{u^{\prime}, v^{\prime}\right\}$ respectively span $D_{0}, D_{1}$ and $D_{2}$, where the indices of $D_{0}$ and $D_{2}$ are both even. The $\operatorname{arcs} \gamma_{0} \cup \gamma_{2}$ and $\gamma_{1}$ agree along a piecewise geodesic subarc $c_{1}$ broken at $p$ and pointing to the directions of $v$ and $u^{\prime}$. We shorten $c_{1}$ in $D_{1}$ into $c_{1}^{\prime}$ while keeping its endpoints fixed by smoothing it out at $p$. The simple closed curve $\gamma_{1}^{\prime}=\left(\gamma_{1} \backslash c_{1}\right) \cup c_{1}^{\prime}$ converges to $\alpha_{1}$ along a path $\gamma_{1, t}^{\prime}$ of 1-cycles with $L\left(\gamma_{1, t}^{\prime}\right) \leq L\left(\gamma_{1}^{\prime}\right)$.

Suppose that there are at least three domains of even index. Let us consider the homotopy of 1-cycles

$$
\gamma_{0, t}+\gamma_{2, t}+\sum_{i \in I^{+} \backslash\{0,2\}} \alpha_{i}
$$

starting from $\sum_{i \in I^{+}} \alpha_{i}$ and joining $\gamma_{0}+\gamma_{2}+\sum_{i \in I^{+} \backslash\{0,2\}} \alpha_{i}$. We extend it by deforming first the subarc $c_{1}$ of $\gamma_{0} \cup \gamma_{2}$ into $c_{1}^{\prime}$, then the curves $\alpha_{i}$ into $\gamma_{i}$ through $\gamma_{i, t}$ for $i \in I^{+} \backslash\{0,2\}$. We get a 1-cycle which agrees with $-\gamma_{1}^{\prime}-\sum_{i \in I^{-} \backslash\{1\}} \gamma_{i}$. Using the homotopies $\gamma_{1, t}^{\prime}$ and $\gamma_{i, t}$, we deform the 1-cycle so obtained into $-\sum_{i \in I^{-}} \alpha_{i}$.

The above construction defines a one-parameter family of 1-cycles $z_{t}^{\prime}$ which starts at $\sum_{i \in I^{+}} \alpha_{i}$ and ends at $-\sum_{i \in I^{-}} \alpha_{i}$. By construction, the family $z_{t}^{\prime}$ is homotopic to $z_{t}$ and satisfies $\sup _{0 \leq t \leq 1} \operatorname{mass}\left(z_{t}^{\prime}\right)<L(\gamma)$.

Suppose that $D_{0}$ and $D_{2}$ are the only domains of even index. Since $\gamma$ is not a figure-eight curve, $\gamma_{0}$ and $\gamma_{2}$ cannot be both geodesic loops based at $p$. We shall assume that $\gamma_{0}$ is not a geodesic loop. Let $c_{0}$ be a piecewise geodesic subarc of $\gamma_{0}$ broken at some point $q \neq p$. We shorten $c_{0}$ in $D_{0}$ into $c_{0}^{\prime}$ by smoothing it out at $q$. We extend the homotopy of 1-cycles $\gamma_{0, t}+\alpha_{2}$ starting from $\alpha_{0} \cup \alpha_{2}$ and joining $\gamma_{0} \cup \alpha_{2}$ by deforming the subarc $c_{0}$ of $\gamma_{0}$ into $c_{0}^{\prime}$. We perturb the 1-cycle so obtained by deforming first the subarc $c_{1}$ into $c_{1}^{\prime}$, then the subarc $c_{0}^{\prime}$ into $c_{0}$. At this stage, we get a 1-cycle which agrees with $-\gamma_{1}^{\prime}-\sum_{i \in I^{-} \backslash\{1\}} \gamma_{i}$. The latter deforms into $-\sum_{i \in I^{-}} \alpha_{i}$, as previously. The 
one-parameter family of 1-cycles $z_{t}^{\prime}$ so defined is homotopic to $z_{t}$ with the same endpoints and satisfies $\sup _{0 \leq t \leq 1} \operatorname{mass}\left(z_{t}^{\prime}\right)<L(\gamma)$.

We can now established Theorem 4.3.

Proof of Theorem 4.3. - Let $\gamma$ be a 1-cycle of mass $\kappa=L_{1}^{\prime}(M)$ which induces a stationary varifold as in Remark 4.1. At least one connected component of $\gamma$ is not a local minimum of the mass functional. From Lemma 4.6 and Lemma 2.3, this component is either a simple closed geodesic of positive index or a figureeight geodesic. Thus, from Lemma 4.5, $\gamma$ is connected and agrees with either a simple closed geodesic of index 1 or a figure-eight geodesic of null index. From the proof of Lemma 4.5 , there exists a homotopy $\gamma_{t}$ in $\mathcal{Z}_{1}^{<\kappa}(M) \cup\{\gamma\}$ which induces a nontrivial class in $\pi_{1}\left(\mathcal{Z}_{1}^{\leq \kappa}(M), \mathcal{Z}_{1}^{\leq \kappa_{0}}(M)\right)$ for some $\kappa_{0}<\kappa$. In particular, the group $\pi_{1}\left(\mathcal{Z}_{1}^{<\kappa}(M) \cup\{\gamma\}, \mathcal{Z}_{1}^{<\kappa}(M)\right)$ is nontrivial. Therefore, $\operatorname{ind}_{\mathcal{Z}_{1}}(\gamma)=1$ and $L_{1}^{\prime \prime}(M) \leq L_{1}^{\prime}(M)$. Moreover, $L_{1}^{\prime \prime}(M)$ agrees with the length of the shortest curve among the simple closed geodesics of index 1 and the figure-eight geodesic of null index.

Suppose that $L_{1}^{\prime \prime}(M)<L_{1}^{\prime}(M)$. Let $\gamma$ be a 1-cycle of index 1 such that $\operatorname{mass}(\gamma)<L_{1}^{\prime}(M)$. As previously, at least one connected component of $\gamma$ is not a local minimum of the mass functional. Therefore, from Lemma 4.6, Lemma 2.3 and Lemma 4.5, the length of this connected component is $\geq L_{1}^{\prime}(M)$. Hence a contradiction.

4.2. Filling radius and the minimal 1-cycle of index 1. - An admissible geodesic on the sphere $M$ is by definition a nontrivial simple closed geodesic of length $\leq \frac{1}{2} L_{1}^{\prime}(M)$ and of null index as geodesic. From Lemma 2.3, an admissible geodesic is also a local minimum of the mass functional. Note that admissible geodesics exist on $M$ if and only if $L_{0}(M) \leq \frac{1}{2} L_{1}^{\prime}(M)$ (we refer to Section 3.2 for the definition of $L_{0}(M)$ ).

An admissible geodesic, which divides $M$ into two disks $D$ and $D^{\prime}$, is said to be extremal with respect to $D$ if no other admissible geodesic lies in $D$.

LEMMA 4.7. - Let $\gamma$ be an admissible geodesic of $M$. Every simple geodesic arc of length $\leq \frac{1}{2} L_{1}^{\prime}(M)$ with endpoints in $\gamma$ is a subarc of $\gamma$.

Proof. - Let us suppose that there exists a simple geodesic arc $c$ of length $\leq \frac{1}{2} L_{1}^{\prime}(M)$ with endpoints in $\gamma$ which lies in the interior of $D$. The arc $c$ divides $D$ into two convex domains $D_{1}$ and $D_{2}$ with $L\left(\partial D_{1}\right)+L\left(\partial D_{2}\right) \leq L_{1}^{\prime}(M)$. The simple closed curves $\partial D_{1}$ and $\partial D_{2}$ converge in the convex domains $D_{1}$ and $D_{2}$ to simple closed geodesics disjoint from $\gamma$ and from each other by the curve-shortening process. The sum of the homotopies so defined is a oneparameter family of 1-cycles $z_{t}$. This family joins two different local minima of the mass functional and satisfies $\operatorname{mass}\left(z_{t}\right)<L_{1}^{\prime}(M)$. We get therefore a contradiction with Claim 4.2. 
Now, we can prove

TheOREM 4.8. - Let $M$ be a bumpy Riemannian 2-sphere, then

$$
\operatorname{FillRad}(M) \geq \frac{1}{20} L_{1}^{\prime}(M)
$$

where $L_{1}^{\prime}(M)$ is the mass of the shortest 1 -cycle of index 1 on $M$.

Proof. - We can assume that there exists an admissible geodesic, otherwise Theorem 3.4 immediately yields the result. Since there are finitely many closed geodesics of length uniformly bounded, there exists an admissible geodesic $\gamma_{0}$ which divides $M$ into two connected components $D$ and $D^{\prime}$ extremal with respect to $D$.

We argue as in the proof of Theorem 3.4. Fix $\delta>0$. Let $P$ be a 3dimensional polyhedron in $U_{\delta}(M)$ containing $M$ as a subpolyhedron. We show that if $\delta<\frac{1}{20} L_{1}^{\prime}(M)$, the inclusion $M \hookrightarrow P$ admits a retraction. Therefore, $M$ does not bound in $U_{\delta}(M)$.

We subdivide $P$ and define a map $r: P^{1} \rightarrow M$ on the 1-skeleton of $P$ which leaves fixed the edges of $M$ as in the proof of Theorem 3.4. The map $r$ takes the edges of $P$ to segments of $M$. Slightly deforming $P$ and $r$ if necessary, we can further assume that no vertex of $P$ is sent to $\gamma_{0}$. Since $i: M \hookrightarrow U_{\delta}(M)$ preserves the distances, the length of the images of the edges of $P$ are bounded from above by $\rho$ with $\rho<\frac{1}{10} L_{1}^{\prime}(M)$. Therefore, the images of the edges of $P$ cut $\gamma_{0}$ at most once from Lemma 4.7. In particular, the images of the boundaries of the 2-simplices of $P$ cut $\gamma_{0}$ at most twice. On every edge of $P$ whose image cuts $\gamma_{0}$, we introduce a new vertex given by the pre-image of the intersection with $\gamma_{0}$. A 3-simplex $\Delta^{3}$ of $P$ having new vertices has exactly three or four. Every new vertex divides the edges where it lies into two new edges. Every pair of new vertices lying in a same face defines a new edge. These three or four new edges of $\Delta^{3}$ bound a new face in $\Delta^{3}$. This defines a new simplicial structure on $P$ modeled on three kinds of building blocks and not only on standard simplices anymore. These building blocks are given by truncating the standard simplex along a plane which does not pass through a vertex.

The intersections of the images of $\partial \Delta^{2}$ with $D$, where $\Delta^{2}$ is a face of the original simplicial structure of $P$, form a finite collection of curves, noted $\mathcal{C}$. The curves of $\mathcal{C}$ are either simple arcs of $D$ with endpoints in $\partial \Delta^{2}$ or simple closed curves of $D$.

Let $p$ and $q$ be two new vertices of $P$ lying in the same face $\Delta^{2}$ of the original simplicial structure of $P$. The intersection of the image of $\partial \Delta^{2}$ with $D$ form a simple arc $\gamma_{p q}$ with endpoints in $\gamma_{0}$ The endpoints, $\bar{p}$ and $\bar{q}$, of $\gamma_{p q}$ are the images by $r$ of $p$ and $q$. We apply the curve-shortening process to the collection $\mathcal{C}$. The arc $\gamma_{p q}$ converges to a simple geodesic arc $\overline{p q}$ of $D$ with endpoints $\bar{p}$ and $\bar{q}$. Since $L\left(\partial \Delta^{2}\right)<\frac{1}{2} L_{1}^{\prime}(M)$, this arc lies in $\gamma_{0}$ from Lemma 4.7. We extend the map

TOME $132-2004-\mathrm{N}^{\mathrm{O}} 1$ 
$r$ on the 1-skeleton $P^{1}$ of the new simplicial structure of $P$ sending the new edges $[p, q]$ onto the arcs $\overline{p q}$ of $\gamma_{0}$.

We now want to extend $r$ to $P^{2}$. Let $\Delta^{2}$ be a face of a building block of $P$. When $\Delta^{2}$ lies in $M$, we fill the boundary $\partial \Delta^{2}$ as in the proof of Theorem 3.4. If the image of $\partial \Delta^{2}$ lies in $D^{\prime}$, we fill it by the disk it bounds in $D^{\prime}$. Otherwise, it lies in $D$. In this case, if $\partial \Delta^{2}$ has an edge which does not lie in the original simplicial structure of $P$, the image of $\partial \Delta^{2}$ retracts onto the image of this edge by construction. If it does not, its image, of length $<\frac{1}{2} L_{1}^{\prime}(M)$, contracts either into an admissible geodesic of $D$, that is, $\gamma_{0}$, which is homotopic to a point in $D^{\prime}$, or into a point in $D$.

In conclusion, we get a map of degree one from $\Delta^{2}$ onto its image in $M$ which agrees with $r$ on the boundary. This yields an extension $r: P^{2} \rightarrow M$ whose restriction to $M$ is the identity.

Let us extend it to $P^{3}$. The restriction of $r: P^{2} \rightarrow M$ to the boundary $\Sigma$ of a building block $\Delta^{3}$ of $P$ is noted $\varphi: \Sigma \rightarrow M$. When the image of $\Sigma$ lies in $D$ or $D^{\prime}$, it extends to a map defined on $\Delta^{3}$ whose image lies in $D$ or $D^{\prime}$. In the general case, the image of $\Sigma$ lies in $M$ and induces a class $[\Sigma]$ in $H_{2}\left(M, D^{\prime}\right) \simeq H_{2}(D, \partial D)$. The map $\varphi$ extends to $\Delta^{3} \rightarrow M$ if and only if $[\Sigma]$ vanishes in $H_{2}(D, \partial D)$. Since the image of the edges of $\Sigma$ are minimizing segments, the image of the 1-skeleton of $\Sigma$ is isotopic to one of the following graphs when it is not degenerated. Note that we can always assume it is the case by slightly perturbing the map $r$.

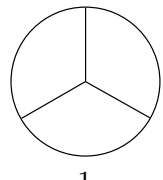

1

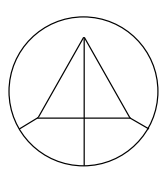

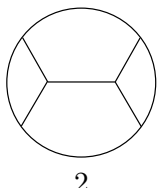

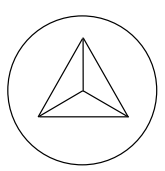

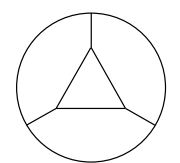

3

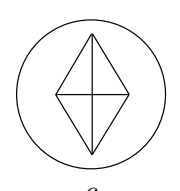

6

Graphs 1-6

In the three following cases, $\Sigma$ is identified with its image by $\varphi$.

Case 1. - Suppose that only one vertex of $\Sigma$ maps in the interior of $D$ (see graph 1). Let $c_{1}, c_{2}$ and $c_{3}$ be the three edges of $\Sigma$ based at this vertex and $\alpha_{1}$, $\alpha_{2}$ and $\alpha_{3}$ be the other edges of $\Sigma$, lying in $\partial D$, with the same endpoints as $c_{2} \cup c_{3}, c_{3} \cup c_{1}$ and $c_{1} \cup c_{2}$. By construction, these three latter arcs are simple and respectively converge to $\alpha_{1}, \alpha_{2}$ and $\alpha_{3}$ through homotopies given by the curve-shortening process. The sum of these homotopies defines a one-parameter 
family $z_{t}$ of 1 -cycles of $D$ which represents $[\Sigma]$. From the property (iv) of the curve-shortening process (see Theorem 2.7), the family $z_{t}$, which starts from a null-current, ends at a 1-cycle lying in $\partial D$ representing either a nullcurrent or $\partial D$. Since mass $\left(z_{t}\right) \leq 6 \rho<L_{1}^{\prime}(M)$, this 1-cycle represents a nullcurrent, otherwise $\gamma_{0}$ would not be a local minimum of the mass functional from Claim 4.2. Therefore, $[\Sigma]=0$ in $H_{2}(D, \partial D)$.

Suppose now that only two vertices of $\Sigma$ map in the interior of $D$ (see graph 2). We argue in the same way. We construct as previously a one-parameter family of 1 -cycles $z_{t}$ in $D$ representing $[\Sigma]$ with $\operatorname{mass}\left(z_{t}\right) \leq 10 \rho<L_{1}^{\prime}(M)$. This homotopy, starting from a null-current, ends also at a null-current. Therefore, it induces a trivial class in homology, that is, $[\Sigma]=0$ in $H_{2}(D, \partial D)$.

Case 2. - Suppose that exactly three vertices of $\Sigma$ map in the interior of $D$ (see graphs 3 and 4). They define a face $\Delta^{2}$ of $\Sigma$. The edges of the boundary $\partial \Delta^{2}$ are noted $e_{1}, e_{2}$ and $e_{3}$. Let $c_{1}, c_{2}$ and $c_{3}$ be the three edges of $\Sigma$ which join the vertices of $\Delta^{2}$ to $\partial D$ (we recall that $\Sigma$ is identified with its image by $\varphi$ ). By construction, the $\operatorname{arcs} c_{2} \cup e_{1} \cup c_{3}, c_{3} \cup e_{2} \cup c_{1}$ and $c_{1} \cup e_{3} \cup c_{2}$ are simple and converge to the edges of $\Sigma$ lying in $\partial D$ through homotopies given by the curve-shortening process. The sum of these homotopies defines a one-parameter family of 1-cycles $z_{t}$ in $D$. This family starts from $\partial \Delta^{2}$ and ends at a 1-cycle lying in $\partial D$ representing either a null-current or $\partial D$ from Theorem 2.7 (iv).

Suppose that it ends at a null-current. Since simple closed curves remain simple through the curve-shortening process, $\partial \Delta^{2}$ converges either into a point or to $\partial D$. This homotopy extends the one-parameter family $z_{t}$. Since mass $\left(z_{t}\right) \leq$ $9 \rho<L_{1}^{\prime}(M)$, we conclude as previously that $[\Sigma]=0$.

Suppose that the family $z_{t}$ ends at $\partial D$. If $\partial \Delta^{2}$ contracts into a point, we extend $z_{t}$ and conclude similarly. Otherwise, $\partial \Delta^{2}$ is homotopic to $\partial D$ by the curve-shortening process. The sum of this homotopy with $z_{t}$, endowed with the orientations induced by the one on $\Sigma$, defines a new one-parameter family of 1-cycles. Since the orientations of the homotopies are induced by the one on $\Sigma$, this new one-parameter family starts at a null-current. Furthermore, since simple curves remain simple through the curve-shortening process, it also ends at a null-current. Thus, the class $[\Sigma]$ it represents vanishes in $H_{2}(D, \partial D)$.

Case 3. - Suppose that the four vertices of $\Sigma$ map in the interior of $D$ (see graphs 5 and 6 ). The boundaries of the faces of $\Sigma$ lie in the interior of $D$ and converge either to points or to $\partial D$ through homotopies $z_{i}^{t}$ given by the curve-shortening process. The faces of $\Sigma$ decompose into two pairs such that the edges of the faces of each pair do not intersect transversely (see graphs 5 and 6). Since the images by the curve-shortening process of simple curves which do not cut each other do not cross (see Theorem 2.7 (iv)), at most two homotopies $z_{i}^{t}$ converge to $\partial D$. 
Suppose that $z_{1}^{t}, z_{2}^{t}$ and $z_{3}^{t}$ converge to points. The one-parameter family of 1-cycles $z_{t}$ formed of $z_{1}^{t}+z_{2}^{t}+z_{3}^{t}$ and $z_{4}^{t}$ starts from a null-current and ends either at a null-current or at $\partial D$. Since mass $\left(z_{t}\right) \leq 9 \rho<L_{1}^{\prime}(M)$, we conclude as previously that the class $[\Sigma]$ it represents vanishes in $H_{2}(D, \partial D)$.

Suppose that $z_{1}^{t}$ and $z_{2}^{t}$ converge to $\partial D$. Since the orientations of the homotopies are induced by the one on $\Sigma$, their sum ends at a null-current. Thus, the one-parameter family of 1 -cycles $z_{1}^{t}+z_{2}^{t}+z_{3}^{t}+z_{4}^{t}$, which represents $[\Sigma]$, starts and ends at null-currents. This implies that $[\Sigma]=0$.

In conclusion, we have extended $r$ on each building block of $P$. This defines a retraction $r: P \rightarrow M$. Hence the result.

Theorems 4.3 and 4.8 immediately lead to the Main Theorem.

REMARK 4.9. - Let $\mathcal{D}$ be the set of disks of $M$ with geodesic boundary containing no nontrivial simple closed geodesic of null index in their interior. The shortest 1-cycles of index 1 of $D$ form a collection of curves when $D$ runs in $\mathcal{D}$. The proof of Theorem 4.8 shows that the mass of the longest of these 1-cycles provides a lower bound on the filling radius. This statement yields a better estimate on the filling radius. Indeed, some small 1-cycles of index 1 can be ignored in the case, for instance, of a sphere with small bumps looking like mushrooms.

REMARK 4.10. - We show in the following examples that it is not possible to restrict the set of curves considered in Theorem 4.8 to a single type of geometry.

The round sphere has no non-simple closed geodesic. Therefore, the length of the shortest simple closed geodesic of index 1 provides in this example the lower bound on the filling radius.

Three long tubes capped with half-hemispheres glued along the boundary components of a hyperbolic pant forms a sphere. This sphere can be pertubed into a bumpy three-leg sphere with arbitrarily small area such that the simple closed geodesics and the closed geodesics of index 1 are arbitrarily long. In this example of a sphere with three long spikes, the length of the shortest figureeight geodesic of null index provides the lower bound on the filling radius.

\section{Filling radius and the global minimax process}

In the previous section, we showed that the lowest minimax value of the local minima processes described in $\left(\mathrm{C}^{\prime} 1\right)-\left(\mathrm{C}^{\prime} 2\right)$ provides a lower bound on the filling radius of the 2 -sphere. However, the minimax value corresponding to the global minimax process described in $(\mathrm{C} 1)-(\mathrm{C} 2)$ of Section 2.1 does not provide any lower bound on the filling radius. The goal of this section is to establish this result by constructing a sequence of metrics on the 2-sphere. More precisely, we have 
THEOREM 5.1. - There exists a sequence $g_{n}$ of Riemannian metrics on $S^{2}$ which satisfies

$$
\lim _{n \rightarrow \infty} \frac{\operatorname{FillRad}\left(S^{2}, g_{n}\right)}{L_{1}\left(S^{2}, g_{n}\right)}=0 .
$$

Let us construct a sequence of metrics $g_{n}$ satisfying the above theorem.

Fix $\varepsilon>0$. Three copies $S, S^{\prime}$ and $S^{\prime \prime}$ of the circle $\mathbb{R} / 2 \varepsilon \mathbb{Z}$ are said to be glued together if the following identifications hold

- $x \in S$ and $\varepsilon-x \in S^{\prime}$ are identified for $-\varepsilon \leq x \leq 0$;

- $x \in S$ and $x \in S^{\prime \prime}$ are identified for $0 \leq x \leq \varepsilon$;

- $x \in S^{\prime}$ and $\varepsilon-x \in S^{\prime \prime}$ are identified for $0 \leq x \leq \varepsilon$.

Fix $n \in \mathbb{N}^{*}$. The quotient of the strip $\left\{(x, y) \in \mathbb{R}^{2} \mid-n \leq y \leq n\right\}$ by the subgroup of displacements $\Gamma$ generated by the translation of vector $(2 \varepsilon, 0)$ defines a flat rectangle cylinder $C$. The curves $\sigma_{y}=\{(x, y) \mid x \in \mathbb{R}\} / \Gamma$ where $-n \leq y \leq n$ are called sections of $C$. The connected components of $\partial C$ agree with $C^{+}=\sigma_{n}$ and $C^{-}=\sigma_{-n}$. The cylinder $C$ decomposes into two semi-cylinders $\left\{\sigma_{y} \mid-n \leq y<0\right\}$ and $\left\{\sigma_{y} \mid 0 \leq y \leq n\right\}$.

We define by induction the cylindrical tree of height $n$ for every $n \in \mathbb{N}^{*}$. The cylindrical tree of height 1 is the flat rectangle cylinder $C$. Its basis is $C^{-}$. The cylindrical tree of height $n+1$ is defined by gluing together the basis of two copies of cylindrical trees of height $n$ with the boundary $C^{+}$of $C$. The boundary $C^{-}$of $C$ forms the basis of the cylindrical tree of height $n+1$.

The tubes modeled on $C$ which form the cylindrical tree $T_{n}$ of height $n$ are noted $M_{i}$ with $i \in I$. The union of $M_{i}$ and the semi-cylinders of $T_{n}$ adjacent to $M_{i}$ is noted $\mathcal{U} M_{i}$.

Let $M$ be the Riemmanian sphere $\left(S^{2}, g_{n}\right)$ obtained by gluing round hemispheres of circumference $2 \varepsilon$ along the connected components of the boundary of $T_{n}$. It is clear that $\operatorname{FillRad}\left(S^{2}, g_{n}\right) \leq \varepsilon$. Let us show that the sequence $L_{1}\left(S^{2}, g_{n}\right)$ is unbounded.

Let $\left(z_{t}\right)_{0 \leq t \leq 1}$ be a one-parameter family of 1-cycles on $M$ satisfying the conditions $(\mathrm{C} 1)-(\mathrm{C} 2)$ of Section 2.1. The truncated family $z_{s}$ with $0 \leq s \leq t$ induces a 2-current $S_{t}$ with $\partial S_{t}=z_{t}$ and $\partial \operatorname{Supp}\left(S_{t}\right) \subset \operatorname{Supp}\left(z_{t}\right)$ (see [2] and [34, $\S$ III.1.4]). The one-parameter family $S_{t}$ is continuous.

We define the index of a tube at the time $t$ by $N_{t}^{i}$ where

$N_{t}^{i}=\left\{\begin{array}{l}1 \text { if there is a simple closed curve of } M_{i} \text { which lies in the interior, } \\ \text { of } \operatorname{Supp}\left(S_{t}\right) \text { and induces a nontrivial class in } H_{1}\left(M_{i}\right) \\ 0 \text { otherwise. }\end{array}\right.$

For $n$ large enough, we can assume that $\operatorname{mass}\left(z_{t}\right)<n$ for every $0 \leq t \leq 1$, otherwise the result is immediate.

At this stage, we need the three following lemmas.

TOME $132-2004-\mathrm{N}^{\mathrm{O}} 1$ 
LEMMA 5.2. - Let $M_{i}$ and $M_{j}$ be two adjacent tubes such that $N_{t}^{i}=1$ and $N_{t}^{j}=0$ with $t$ fixed. Then, there is an irreductible component, i.e., a simple closed curve, of $\operatorname{Supp}\left(z_{t}\right) \cap \mathcal{U} M_{i}$ homotopic in $T_{n}$ to a section of $M_{i}$ or $M_{j}$.

Proof. - Assume that no irreductible component of $\operatorname{Supp}\left(z_{t}\right) \cap \mathcal{U} M_{i}$ is homotopic to a section of $M_{j}$ in $T_{n}$. Let $M_{k}$ be the tube adjacent to both $M_{i}$ and $M_{j}$. Let $c$ be a simple closed curve of $M_{i}$ lying in the interior of $\operatorname{Supp}\left(S_{t}\right)$ and representing a nontrivial class in $H_{1}\left(M_{i}\right)$. The connected component of $\operatorname{Supp}\left(S_{t}\right)$ containing $c$ is noted $\operatorname{Supp}_{0}\left(S_{t}\right)$. Recall that we have supposed mass $\left(z_{t}\right)<n$. Therefore, from the coarea inequality, there exist $c_{j}$ and $c_{k}$ in $\mathcal{U} M_{i}$, sections of $M_{j}$ and $M_{k}$, which do not intersect $\operatorname{Supp}\left(z_{t}\right)$. In particular, $c_{j}$ is disjoint from $\partial \operatorname{Supp}\left(S_{t}\right)$. Therefore, either $c_{j}$ lies in $\operatorname{Supp}\left(S_{t}\right)$ (which is impossible since $\left.N_{t}^{j}=0\right)$ or $c_{j}$ is disjoint from $\operatorname{Supp}\left(S_{t}\right)$.

Let $\Upsilon \subset \mathcal{U} M_{i}$ be the connected domain bounded by $c, c_{j}$ and $c_{k}$. Let $\operatorname{Supp}_{0}\left(S_{t}\right)$ be the connected component of $\operatorname{Supp}\left(S_{t}\right)$ containing $c$. The curve $c$ is homologuous in $\Upsilon_{t}:=\operatorname{Supp}_{0}\left(S_{t}\right) \cap \Upsilon$ to $\partial \Upsilon_{t} \backslash c$. We have

$$
\partial \Upsilon_{t} \backslash c \subset\left[\operatorname{Supp}\left(z_{t}\right) \cap \Upsilon\right] \amalg\left[\left(\operatorname{Supp}_{0}\left(S_{t}\right) \cap \partial \Upsilon\right) \backslash c\right],
$$

where the union is disjoint. Since $c_{j}$ is disjoint from $\partial \operatorname{Supp}_{0}\left(S_{t}\right)$ and $N_{t}^{j}=0$, the term $\left(\operatorname{Supp}_{0}\left(S_{t}\right) \cap \partial \Upsilon\right) \backslash c$ is either the empty set or $c_{k}$. By assumption, no irreductible component of $\operatorname{Supp}\left(z_{t}\right) \cap \mathcal{U} M_{i}$ is homotopic to $c_{j}$ in $T_{n}$. Therefore, the irreductible components of the term $\operatorname{Supp}\left(z_{t}\right) \cap \Upsilon$ are either homotopically trivial in $T_{n}$ or homotopic to $c$ or $c_{k}$ in $T_{n}$. In conclusion, there is an irreductible component of $\operatorname{Supp}\left(z_{t}\right) \cap \mathcal{U} M_{i}$ homotopic to a section of $M_{i}$ in $T_{n}$, otherwise the curve $c$ would be homologuous to $c_{k}$ in $T_{n}$, which is absurd.

We define by induction the height of a tube on a cylindrical tree $T_{n}$. The tube at the basis of $T_{n}$ is at the height zero. Every tube adjacent to a tube of height $k$ which is not itself of height $\leq k$ is by definition of height $k+1$. The height of a tube $M_{i} \subset T_{n}$, noted $h\left(M_{i}\right)$, satisfies $0 \leq h\left(M_{i}\right) \leq n-1$.

Define $N_{t}$ as the cardinal of

$$
A_{t}=\left\{(i, j) \mid M_{i} \text { and } M_{j} \text { are adjacent with } N_{t}^{i}=1 \text { and } N_{t}^{j}=0\right\} .
$$

LEMMA 5.3. - Let $0 \leq t \leq 1$. We have $\operatorname{mass}\left(z_{t}\right) \geq \frac{1}{8} N_{t} \varepsilon$.

Proof. — The set $A_{t}$ decomposes into the disjoint union of

$$
A_{t}^{\prime}=\left\{(i, j) \in A_{t} \mid h\left(M_{i}\right) \text { is odd }\right\} \quad \text { and } \quad A_{t}^{\prime \prime}=\left\{(i, j) \in A_{t} \mid h\left(M_{i}\right) \text { is even }\right\} .
$$

Define the projection $\pi$ on the first coordinate by $\pi(i, j)=i$. It is possible to divide $A_{t}^{\prime}$ into two subsets whose $\pi$-projections contain no pair $\left\{i, i^{\prime}\right\}$ such that $M_{i}$ and $M_{i^{\prime}}$ are adjacent. The same goes for $A_{t}^{\prime \prime}$. In this way, the set $A_{t}$ decomposes into four disjoint subsets. One of them, noted $B_{t}$, contains at least $\frac{1}{4} N_{t}$ elements. From Lemma 5.2, there exists an irreductible component of 
$\operatorname{Supp}\left(z_{t}\right) \cap \mathcal{U} M_{i}$ of length $\geq 2 \varepsilon$ for every $i \in \pi\left(B_{t}\right)$. By construction, the neighborhoods $\mathcal{U} M_{i}$ where $i \in \bar{\pi}\left(B_{t}\right)$ are disjoint. Therefore, $\operatorname{mass}\left(z_{t}\right) \geq 2\left|\pi\left(B_{t}\right)\right| \varepsilon$. But $\left|\pi\left(B_{t}\right)\right| \geq \frac{1}{4}\left|B_{t}\right| \geq \frac{1}{16} N_{t}$, hence the result.

LEMMA 5.4. - Let $0 \leq t \leq 1$. The number of tubes of $M$ of index 1 at the time $t$ can be written under the form $\sum_{i \in S} \epsilon_{i}\left(2^{n_{i}}-1\right)$ where $S \subset\left\{0, \ldots, N_{t}\right\}$, $\epsilon_{i}= \pm 1$ and $1 \leq n_{i} \leq n$. In particular, there are at most $(2 n)^{N_{t}+1}$ such numbers.

Proof. - We follow the proof of [15, Lemma 6]. Define

$B_{t}^{\prime}=\left\{(i, j) \in A_{t} \mid h\left(M_{i}\right)>h\left(M_{j}\right)\right\}$ and $B_{t}^{\prime \prime}=\left\{(i, j) \in A_{t} \mid h\left(M_{i}\right)<h\left(M_{j}\right)\right\}$.

The tube $M_{i} \subset T_{n}$ is the basis of a unique cylindrical tree of maximal height $n-h\left(M_{i}\right)$ contained in $T_{n}$. Given $k \in \mathbb{N}$, let $\tau_{k}^{+}$be the union of the cylindrical trees of $T_{n}$ of basis $M_{i}$ and of maximal height with $h\left(M_{i}\right)=k$ and $(i, j) \in B_{t}^{\prime}$ for some $j$. For $k=0, \tau_{0}^{+}$agrees with the empty set if the index of the basis of $T_{n}$ is null or with $T_{n}$ otherwise. Similarly, let $\tau_{k}^{-}$be the union of the cylindrical trees of $T_{n}$ of basis $M_{j}$ and of maximal height with $h\left(M_{i}\right)=k$ and $(i, j) \in B_{t}^{\prime \prime}$ for some $j$.

The set of the tubes of $M$ of index 1 at the time $t$ agrees with the disjoint union $\left(\ldots\left(\left(\left(\tau_{0}^{+} \backslash \tau_{0}^{-}\right) \amalg \tau_{1}^{+}\right) \backslash \tau_{1}^{-}\right) \amalg \tau_{2}^{+} \cdots \backslash \tau_{n-2}^{-}\right) \amalg \tau_{n-1}^{+}$. Therefore, the number of the tubes of $M$ of index 1 at the time $t$ equals

$$
\sum_{(i, j) \in B_{t}^{\prime}}\left(2^{n-h\left(M_{i}\right)}-1\right)-\sum_{(i, j) \in B_{t}^{\prime \prime}}\left(2^{n-h\left(M_{j}\right)}-1\right)
$$

if the index of the basis of $T_{n}$ is null and

$$
\sum_{(i, j) \in B_{t}^{\prime}}\left(2^{n-h\left(M_{i}\right)}-1\right)-\sum_{(i, j) \in B_{t}^{\prime \prime}}\left(2^{n-h\left(M_{j}\right)}-1\right)+2^{n}-1
$$

otherwise. In both cases, it is of the desired form.

Let us show how to conclude from the previous lemmas.

Slightly perturbing the homotopy $z_{t}$ if necessary, we can suppose that the indices $N_{t}^{i}$ change one at a time throughout the homotopy. Note that the small perturbations used do not increase the mass of the 1-cycles $z_{t}$ more than any preliminary given number. When $t$ varies from 0 to 1 , the number of tubes of $M$ of index 1 varies from 0 at $t=0$ to $2^{n}-1$ at $t=1$. Thus, each integer between 0 and $2^{n}-1$ occurs as the number of tubes of $M$ of index 1 at some time $t$. Therefore, we have $(2 n)^{N_{t}+1} \geq 2^{n}$ for some $t$, from Lemma 5.4. That is, $N_{t}$ is bigger than $c n / \ln n$ for some $t$ where $c$ is a positive constant independent of $n$ and $t$. In particular, we get $\sup _{0 \leq t \leq 1} \operatorname{mass}\left(z_{t}\right) \geq c^{\prime} n / \ln n \varepsilon$ with $c^{\prime}>0$ independent of $n$ from Lemma 5.3. Therefore, $L_{1}\left(S^{2}, g_{n}\right)$ is unbounded.

TOME $132-2004-\mathrm{N}^{\mathrm{O}} 1$ 


\section{BIBLIOGRAPHY}

[1] Accola (R.D.M.) - Differential and extremal lengths on Riemannian surfaces, Proc. Math. Acad. Sci. USA, t. 46 (1960), pp. 83-96.

[2] Almgren (F.) - The homotopy groups of the integral cycle groups, Topology, t. 1 (1960), pp. 257-299.

[3] Babenko (I.) - Asymptotic invariants of smooth manifolds, Russian Acad. Sci. Izv. Math., t. 41 (1993), pp. 1-38.

[4] Bangert (V.) \& Katz (M.) - Riemannian manifolds with harmonic 1forms of constant norms, Preprint.

[5] - Stable systolic inequalities and cohomology products, Comm. Pure Appl. Math., t. 56 (2003).

[6] Bavard (C.) - Inégalité isosystolique pour la bouteille de Klein, Math. Ann., t. 274 (1986), pp. 439-441.

[7] Berger (M.) - Systole et applications selon Gromov, in Sémin. Bourbaki, Astérisque, vol. 216, Soc. Math. France, 1993, pp. 279-310.

[8] Blatter (C.) - Über extremallägen auf geschlossen flächen, Comment. Math. Helv., t. 35 (1961), pp. 55-62.

[9] Burago (Yu.) \& Zalgaller (V.) - Geometric inequalities, Springer, 1988.

[10] Calabi (E.) \& CaO (J.) - Simple closed geodesics on convex surfaces, J. Diff. Geom., t. 36 (1992), pp. 517-549.

[11] Cheeger (J.) \& EBin (D.) - Comparaison theorems in Riemannian geometry, North-Holland, Amsterdam, 1975.

[12] Croke (C.) - Area and the length of shortest closed geodesic, J. Diff. Geom., t. 27 (1988), pp. 1-22.

[13] Croke (C.) \& Katz (M.) - Universal volume bounds in Riemannian manifolds, Surveys in Differential Geometry, vol. 8, to appear.

[14] Federer (H.) - Geometric measure theory, Springer-Verlag, New York, 1969.

[15] Frankel (S.) \& Katz (M.) - Morse landscape of a Riemannian disk, Ann. Inst. Fourier, t. 43 (1993), pp. 503-507.

[16] Grayson (M.) - Shortening embedded curves, Ann. Math., t. 129 (1989), pp. $71-111$.

[17] Gromov (M.) - Filling Riemannian manifolds, J. Diff. Geom., t. 18 (1983), pp. 1-147.

[18] __ Systoles and intersystolic inequalities, in Actes de la Table Ronde de Géométrie Différentielle (Luminy, 1992), Sémin. Congr., vol.1, Soc. Math. France, 1996, pp. 291-363.

[19] _ Metric structures for Riemannian and non-Riemannian spaces, Progr. in Mathematics, vol. 152, Birkhäuser, Boston, 1999. 
[20] Hass (J.) \& ScotT (P.) - Shortening curves on surfaces, Topology, t. 33 (1994), pp. 25-43.

[21] Hebda (J.) - Some lower bounds for the area of surfaces, Invent. Math., t. 65 (1982), pp. 485-491.

[22] _ The collars of a Riemannian manifold and stable isosystolic inequalities, Pacific J. Math., t. 121 (1986), pp. 339-356.

[23] KAtz (M.) - The filling radius of two-point homogeneous spaces, J. Diff. Geom., t. 18 (1983), pp. 505-511.

[24] Katz (M.), KRECK (M.) \& SuCIU (A.) - Free abelian covers, short loops, stable length and systolic inequalities, Preprint.

[25] Klingenberg (W.) - Lectures on closed geodesics, Appendix, Grundlehren Math. Wiss., vol. 230, Springer-Verlag, Berlin, 1978.

[26] MaEda (M.) - The length of a closed geodesic on a compact surface, Kyushu J. Math., t. 48 (1994), no. 1, pp. 9-18.

[27] Morgan (F.) - Geometric measure theory. A beginner's guide, 2nd ed., Academic Press, 1995.

[28] Nabutovsky (A.) \& Rotman (R.) - Volume, diameter and the minimal mass of a stationnary 1-cycle, Preprint.

[29] _ The length of the shortest closed geodesic on a 2-dimensional sphere, Int. Math. Res. Not., t. 23 (2002), pp. 1211-1222.

[30] _ Upper bounds on the length of a shortest closed geodesic and quantitative Hurewicz theorem, J. Eur. Math. Soc., t. 5 (2003), pp. 203-244.

[31] PitTs (J.) - Regularity and singularity of one dimensional stational integral varifolds on manifolds arising from variational methods in the large, Symposia Mathematics, vol. XIV, Roma, Italy, 1974.

[32] _ Existence and regularity of minimal surfaces on Riemannian manifolds, Math. Notes, vol. 27, Princeton University Press, 1981.

[33] PU (P.M.) - Some inequalities in certain nonorientable Riemannian manifolds, Pacific J. Math., t. 2 (1952), pp. 55-71.

[34] De Rham (G.) - Differentiable manifolds, Grundlehren Math. Wiss., vol. 266, Springer-Verlag, Berlin, 1984.

[35] Rotman (R.) - Upper bounds on the length of the shortest closed geodesic on simply connected manifolds, Math. Z., t. 233 (2000), pp. 365-398.

[36] SAKAI (T.) - A proof of the isosystolic inequality for the Klein bottle, Proc. Amer. Math. Soc., t. 104 (1988), pp. 589-590.

[37] White (B.) - A strong minimax property of nondegenerate minimal submanifolds, J. reine angew. Math., t. 457 (1994), pp. 203-218.

[38] Wilhelm (F.) - On radius, systole and positive Ricci curvature, Math. Z., t. 218 (1995), pp. 597-602. 